\title{
CLASSIFICATION OF SPECIAL CURVES IN THE SPACE OF CUBIC POLYNOMIALS
}

\author{
by
}

\author{
Charles Favre \& Thomas Gauthier
}

\begin{abstract}
We describe all special curves in the parameter space of complex cubic polynomials, that is all algebraic irreducible curves containing infinitely many post-critically finite polynomials. This solves in a strong form a conjecture by Baker and DeMarco for cubic polynomials.

Let $\operatorname{Per}_{m}(\lambda)$ be the algebraic curve consisting of those cubic polynomials that admit an orbit of period $m$ and multiplier $\lambda$. We also prove that an irreducible component of $\operatorname{Per}_{m}(\lambda)$ is special if and only if $\lambda=0$.
\end{abstract}

\section{Contents}

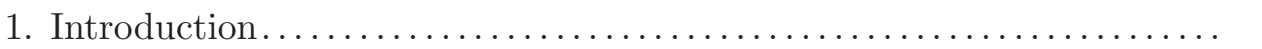

2. The Böttcher coordinate of a polynomial...................

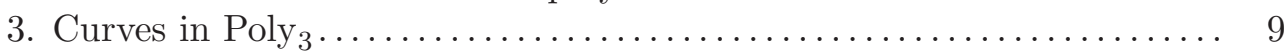

4. Green functions on special curves....................... 17

5. Special curves having a periodic orbit with a constant multiplier..... 21

6. A polynomial on a special curve admits a symmetry............ 26

7. Classification of special curves........................... 29

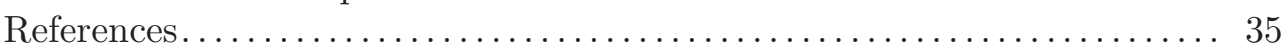

\section{Introduction}

The space Poly ${ }_{d}$ of complex polynomials of degree $d \geq 2$ modulo affine conjugacy forms a complex analytic space that admits a ramified parameterization by the affine space $\mathbb{A}_{\mathbb{C}}^{d-1}$. The study of the set of degree $d$ polynomials with special dynamical features forms the core of the modern theory of holomorphic dynamics. We shall be concerned here with the distribution of the set of post-critically finite $(\mathrm{PCF})$ polynomials for which all critical points have a finite orbit under iteration. This set is a countable union of points defined over a number field, see e.g. II Corollary 3]. It was proved in $\mathbf{L}$ that hyperbolic PCF quadratic polynomials equidistribute to the harmonic measure of the Mandelbrot set. This convergence was generalized in [BH1 in degree 2, and later in [FG] in any degree where it was proved that under a mild assumption any sequence of Galois-invariant finite subsets of PCF polynomials converges in the sense of measures to the so-called bifurcation measure. This fact was further explored in [GV]. The support of this measure has been characterized in several ways in a series of works [DF, Du1, Du2, G3], and it was shown by the second author $[\mathbf{G 1}$ to have maximal Hausdorff dimension $2(d-1)$.

First author is supported by the ERC-starting grant project "Nonarcomp" no.307856, both authors are partially supported by ANR project "Lambda" ANR-13-BS01-0002. 
In a beautiful recent paper [BDM], Baker and DeMarco have proposed a way to describe the distribution of PCF polynomials from the point of view of the Zariski topology. They defined special algebraic subvarieties as those subvarieties $Z \subset$ Poly $_{d}$ admitting a Zariski-dense subset formed by PCF polynomials, and asked about the classification of such varieties. More precisely, they offered a quite general conjecture BDM, Conjecture 1.4] inspired by the André-Oort conjecture in arithmetic geometry stating that any polynomia (1) lying in a special (proper) subvariety should admit a critical orbit relation (see also [Si2, Conjecture 6.56]). They gave a proof of a stronger version of this conjecture in the case the subvariety was isomorphic to an affine line.

Our objective is to give the list of all special curves in the case $d=3$, thereby proving Baker-DeMarco's conjecture for cubic polynomials. The geometry of the space of cubic polynomials has been thoroughly explored in the seminal work $\mathbf{B H 2}$ of Branner and Hubbard. Instead of using their parameterization, we shall follow DF and consider the parameterization $(c, a) \mapsto P_{c, a}$ of the parameter space by the affine plane with

$$
P_{c, a}(z):=\frac{1}{3} z^{3}-\frac{c}{2} z^{2}+a^{3}, z \in \mathbb{C},(c, a) \in \mathbb{C}^{2} .
$$

Observe that $P_{c, a}$ then admits two critical points $c_{0}:=0$ and $c_{1}:=c$ and that this map defines a finite branched cover of the moduli space Poly ${ }_{3}$ of cubic polynomials with marked critical points.

Here is our main result.

Theorem A. - An irreducible curve $C$ in the space $\mathrm{Poly}_{3}$ contains an infinite collection of post-critically finite polynomials if and only if one of the following holds.

1. One of the two critical points is persistently pre-periodic on $C$, i.e. there exist integers $m>0$ and $k \geq 0$ such that $P_{c, a}^{m+k}\left(c_{0}\right)=P_{c, a}^{k}\left(c_{0}\right)$ or $P_{c, a}^{m+k}\left(c_{1}\right)=P_{c, a}^{k}\left(c_{1}\right)$ for all $(c, a) \in C$.

2. There is a persistent collision of the two critical orbits on $C$, i.e. there exist $(m, k) \in$ $\mathbb{N}^{2} \backslash\{(1,1)\}$ such that $P_{c, a}^{m}\left(c_{1}\right)=P_{c, a}^{k}\left(c_{0}\right)$ for all $(c, a) \in C$.

3. The curve $C$ is given by the equation $\left\{(c, a), 12 a^{3}-c^{3}-6 c=0\right\}$, and coincides with the set of cubic polynomials having a non-trivial symmetry, i.e. the set of parameters $(c, a)$ for which $Q_{c}(z):=-z+c$ commutes with $P_{c, a}$.

Recall that for any integer $m \geq 1$ and any complex number $\lambda \in \mathbb{C}$ the set $\operatorname{Per}_{m}(\lambda)$ consisting of all polynomials $P_{c, a} \in$ Poly $_{3}$ that admits at least one periodic orbit of period $m$ and multiplier $\lambda$ is an algebraic curve (see 95 for a more precise description).

The geometry of these curves has been explored by several authors, especially when $\lambda=$ 0 . The irreducible components of $\operatorname{Per}_{m}(0)$ has been proven to be smooth by Milnor [Mi3], and the escape components of these curves have been described in terms of Puiseux series by Bonifant, Kiwi and Milnor [BKM] (see also [K, $\S 7]$ ). On the other hand, DeMarco and Schiff [DMS] have given an algorithm to compute their Euler characteristic.

From the point of view of pluripotential theory, the distribution of the sequence of curves $\left(\operatorname{Per}_{m}(\lambda)\right)_{m \geq 1}$ has been completely described by Bassanelli and Berteloot in [BB2] in the case $|\lambda| \leq 1$ (see also G2 for the case $|\lambda|>1$ and [BB1 for the case of quadratic rational maps).

\footnotetext{
$\overline{(1)}$ the conjecture is actually stated for any rational maps, and a stronger conjecture related to Pink and Zilber's conjectures can be found in De2.
} 
Inspired by a similar result from Baker and DeMarco, see BDM, Theorem 1.1] we also give a characterization of those $\mathrm{Per}_{m}(\lambda)$ that contain infinitely many PCF polynomials. This answers a conjecture of DeMarco in the case of cubic polynomials (see e.g. Si2, Conjecture 6.59]). More precisely, we prove

Theorem B. - For any $m \geq 1$, the curve $\operatorname{Per}_{m}(\lambda)$ contains infinitely many postcritically finite polynomials if and only if $\lambda=0$.

The general strategy of the proof of these two theorems was set up by Baker and DeMarco. They start with an irreducible algebraic curve $C \subset$ Poly $_{3}$ containing infinitely many PCF polynomials (in Theorem B the curve $C$ is a component of some $\operatorname{Per}_{m}(\lambda)$ ). We observe however that they used at several key points their assumption that the curve $C$ has a single branch at infinity. To remove this restriction we had to include two new ingredients:

- we construct a one parameter family of heights for which Thuillier-Yuan's equidistribution theorem $[\mathbf{Y}, \mathbf{T}$ ] applies;

- we investigate systematically the arithmetic properties of the coefficients of the expansion of the Böttcher coordinates and its dependence on the parameters $c, a$.

We propose also a new way to build the symmetry by relying on a recent algebraization result of Xie $[\mathbf{X}]$ that gives a criterion for when a formal curve in the affine plane is a branch of an algebraic curve.

A characteristic feature of our proofs is to look at the dynamics induced by cubic polynomials over various fields: over the complex numbers and over $p$-adic fields (see e.g. \$4.1), over the field of Laurent series (see the proof of Proposition 3.6 and \$5), and over a number field (see 83). We use at one point the universality theorem of McMullen McM3 which is a purely Archimedean statement. Moreover the work of Kiwi $[\mathbf{K}]$ on non-Archimedean cubic polynomials over a field of residual characteristic zero plays a key role in the proof of Theorem B.

Let us describe in more detail how we proceed, and so pick an irreducible algebraic curve $C \subset$ Poly $_{3}$ containing infinitely many PCF polynomials. We may suppose that neither $c_{0}$ nor $c_{1}$ are persistently pre-periodic on $C$. By a theorem of McMullen [McM1, Lemma 2.1] this is equivalent to say that both critical points exhibit bifurcations at some (possibly different) points in $C$. There is a more quantitative way to describe the set of bifurcations using the Green function $g_{c, a}(z):=\lim _{n \rightarrow \infty} \frac{1}{3^{n}} \log \max \left\{1,\left|P^{n}(z)\right|\right\}$. Indeed both functions $g_{0}(c, a):=g_{c, a}\left(c_{0}\right), g_{1}(c, a):=g_{c, a}\left(c_{1}\right)$ are non-negative and pluri-subharmonic, and it is a fact [De1, §5] that the support of the positive measure $\left.\Delta g_{0}\right|_{C}$ (resp. $\left.\Delta g_{1}\right|_{C}$ ) is equal to the set of parameters where $c_{0}$ (resp. $c_{1}$ ) is unstable.

The first step consists in proving that $\left.g_{0}\right|_{C}$ and $\left.g_{1}\right|_{C}$ are proportional, and this conclusion is obtained by applying an equidistribution result of points of small height due to Yuan [Y] and Thuillier $[\mathbf{T}]$. We first observe that $C$ is necessarily defined over a number field $\mathbb{K}$ since it contains infinitely many PCF polynomials, so that we may introduce the functions $g_{0, v}, g_{1, v}$ for all (not necessarily Archimedean) places $v$ over $\mathbb{K}$. These functions can now be used to build a one-parameter family of heights on $C$ by setting

$$
h_{s}(p):=\frac{1}{\operatorname{deg}(p)} \sum_{q, v} \max \left\{s_{0} g_{0, v}(q), s_{1} g_{1, v}(q)\right\}
$$

where $s=\left(s_{0}, s_{1}\right) \in \mathbb{R}_{+}^{2}$, and the sum ranges over all Galois conjugates $q$ of $p$ and over all places $v$ over $\mathbb{K}$. When $s_{0}$ and $s_{1}$ are positive integers, then we prove in $\$ 3$ that the height 
$h_{s}$ is induced by a continuous semi-positive adelic metrization in the sense of Zhang on a suitable line bundle over $C$ of positive degree, so that Thuillier-Yuan's theorem applies. This gives us sufficiently many restrictions on $g_{0}$ and $g_{1}$ which force their proportionality. The key arguments are Proposition 3.6 that is close in spirit to BDM, Proposition 2.1 (3)], and the fact that the function $\max \left\{g_{0, v}, g_{1, v}\right\}$ is a proper continuous function on $\mathrm{Poly}_{3}$ for any place $v$.

From the proportionality of $g_{0}$ and $g_{1}$ on a special curve, we are actually able to conclude the proof of Theorem B. This step is done in \$5. We suppose by contradiction that our special curve $C$ is an irreducible component of some $\operatorname{Per}_{m}(\lambda)$ with $\lambda \neq 0$. Then each branch at infinity of $C$ defines a cubic polynomial over the field of Laurent series $\mathbb{C}((t))$. We show that except when $c_{0}$ or $c_{1}$ is persistently periodic in $C$ the multipliers of all periodic points are exploding on that branch by $[\mathbf{K}]$. We then analyze the situation of a unicritica $(2)$ polynomial in $C$ and computing the norm of the multiplier of its periodic points in a suitable field of residual characteristic 3 , we are able to get the required contradiction.

Let us come back to the proof of Theorem $\mathrm{A}$. At this point, we have an irreducible algebraic curve $C$ defined over a number field $\mathbb{K}$ and such that $g_{0, v}=g_{1, v}$ at any place $v$ over $\mathbb{K}$. Recall that for any polynomial $P_{c, a}$ there exists an analytic isomorphism near infinity conjugating the polynomial to the cubic monomial map. This isomorphism is referred to as the Böttcher coordinate $\varphi_{c, a}$ of $P_{c, a}$. We prove that when $c, a$ are defined over a number field then $\varphi_{c, a}$ is a power series with coefficients in a number field whose domain of convergence is positive at any place, see Lemma 2.2 and Proposition 2.3.

Building on an argument of Baker and DeMarco, we then show that outside a compact subset of the analytification of $C$ (for any completion of $\mathbb{K}$ ) the values of the Böttcher coordinates at $c_{0}$ and $c_{1}$ are proportional up to a root of unity (Theorem 4.1 (2)).

The proof now takes a slight twist as we fix any polynomial $P:=P_{c, a}$ that is not post-critically finite and for which $(c, a)$ belongs to $C(\mathbb{L})$ for some finite field extension $\mathbb{L}$ of $\mathbb{K}$. We prove that any such polynomial admits a weak form of symmetry in the sense that there exists an irreducible curve $Z_{P} \subset \mathbb{P}^{1} \times \mathbb{P}^{1}$ that is stable by the map $(P, P)$. To do so we apply [X] Theorem 1.5] as an alternative to the arguments of Baker and DeMarco in [BDM, §5.6]. In order to get a polynomial that commutes with $P$ instead of a correspondence, we proceed as Baker and DeMarco and use Medvedev-Scanlon's result [MS, Theorem 6.24] (see [ $\mathbf{P}$, Theorem 4.9] for another proof of this result).

At this point we have proved the following result that we feel is of independent interest.

Theorem C. - Pick any irreducible complex algebraic curve $C \subset$ Poly $_{3}$. Then the following assertions are equivalent:

1. the curve $C$ is special,

2. for any critical point that is not persistenly pre-periodic on $C$, the set of PCF polynomials lying in $C$ is equal to the set where this critical point is pre-periodic;

3. the curve $C$ is defined over a number field $\mathbb{K}$ and there exist integers $\left(s_{0}, s_{1}\right) \in$ $\mathbb{N}^{2} \backslash\{(0,0)\}$ such that for any place $v \in M_{\mathbb{K}}$, we have

$$
s_{0} \cdot g_{0, v}=s_{1} \cdot g_{1, v},
$$

on the analytification of $C$ over the completion of $\mathbb{K}$ w.r.t. the $v$-adic norm;

$\overline{{ }^{(2)} \text { i.e. having a }}$ single critical point 
4. the curve $C$ is special, and for any sequence $X_{k} \subset C$ of Galois-invariant finite sets of $P C F$ polynomials with $X_{k} \neq X_{l}$ for $l \neq k$, the probability measures $\mu_{k}$ equidistributed on $X_{k}$ converge towards (a multiple of) the bifurcation measure $T_{\mathrm{bif}} \wedge[C]$ as $k \rightarrow \infty$;

5. there exists a root of unity $\zeta$, and integers $q, m \geq 0$ such that the polynomial $Q_{c, a}(z):=\zeta P_{c, a}^{m}(z)+(1-\zeta) \frac{c}{2}$ commutes with any iterate $P_{c, a}^{k}$ such that $\zeta^{3^{k}}=\zeta$, and $Q_{c, a}\left(P_{c, a}^{q}\left(c_{i}\right)\right)=P_{c, a}^{q}\left(c_{j}\right)$ for some $i, j \in\{0,1\}$ and all $(c, a) \in C$.

In (4) the current $T_{\text {bif }}$ is defined as the $d d^{c}$ of the plurisubharmonic function $g_{0}+g_{1}$. Its support in $\mathbb{C}^{2}$ is known to be equal to the set of unstable parameters, see e.g. [DF, $\S 3]$. Notice that for any curve $C$ there exists a critical point which is not persistently preperiodic on $C$ since by $\left[\mathbf{B H 2}\right.$ the set $\left\{g_{0}=0\right\} \cap\left\{g_{1}=0\right\}$ is compact in $\mathbb{A}_{\mathbb{C}}^{2}$. In particular, the assertion (2) is consistent.

To complete the proof of Theorem $\mathrm{A}$, we analyze in more detail the possibilities for a cubic polynomial to satisfy the condition (5) in the previous theorem. Namely, we prove that the set of parameters admitting a non-trivial symmetry of degree $3^{m}>1$ is actually finite. Theorems $\mathrm{A}$ and $\mathrm{C}$ are proved in $\$ 7$.

We have deliberately chosen to write the entire paper for cubic polynomials only. This simplifies the exposition, but many parts of the proof actually extend to a larger context. Let us briefly discuss the possible extensions and the limitations of our approach.

All ingredients are present to prove Baker-DeMarco's conjecture for a curve in the space of polynomials of any degree $d \geq 2$. It is however not clear to the authors how to obtain the more precise classification of special curves in the same vein as in Theorem $\mathrm{A}$.

We note that there are serious difficulties that lie beyond the methods presented here to handle higher dimensional special varieties $V$ in Poly $_{d}$. The main issue is the following. To apply Yuan's equidistribution theorem of points of small heights it is necessary to have a continuous semi-positive adelic metrics on an ample line bundle on a compactification of $V$, and we are at the moment very far from being able to check any of the three underlined conditions.

Trying to understand special curves in the space of quadratic maps requires much more delicate estimates than in the case of polynomials. A first important step has been done by DeMarco, Wang and Ye in a recent paper [DMWY].

Acknowledgements. - We thank Xavier Buff and Laura DeMarco for discussions at a preliminary stage of this project, and the referee for his/her careful reading of this paper and his/her constructive remarks.

While finishing the writing of this paper we have learned that Dragos Ghioca and Hexi Ye have independently obtained a proof of Theorem A. Their approach differs from ours in the sense that they directly prove the continuity of the metrizations induced by the functions $g_{0, v}$ and $g_{1, v}$. We get around this problem by considering metrizations induced by $\max \left\{s_{0} g_{0, v}, s_{1} g_{1, v}\right\}$ for positive $s_{0}, s_{1}$ instead. We warmly thank D. Ghioca and H. Ye for sharing with us their preprint.

\section{The Böttcher coordinate of a polynomial}

In this section, $K$ is any complete metrized field of characteristic zero containing a squareroot $\omega$ of $\frac{1}{3}$. It may or may not be endowed with a non-Archimedean norm. 
If $X$ is an algebraic variety over $K$, then $X^{\text {an }}$ denotes its analytification as a real-analytic or a complex variety if $K$ is Archimedean, and as a Berkovich analytic space when $K$ is non-Archimedean (see e.g. [B, $§ 3.4-5]$ ).

\subsection{Basics}

As in the introduction, we denote by Poly $_{3} \simeq \mathbb{A}^{2}$ the space of cubic polynomials defined by

$$
P_{c, a}(z):=\frac{1}{3} z^{3}-\frac{c}{2} z^{2}+a^{3} .
$$

It is a branched cover of the parameter space of cubic polynomials with marked critical points. The critical points of $P_{c, a}$ are given by $c_{0}:=c$ and $c_{1}:=0$.

For a fixed $(c, a) \in K^{2}$ the function $\frac{1}{3} \log ^{+}\left|P_{c, a}(z)\right|-\log ^{+}|z|$ is bounded on $\mathbb{A}_{K}^{1, \text { an }}$ so that the sequence $\frac{1}{3^{n}} \log ^{+}\left|P_{c, a}^{n}(z)\right|$ converges uniformly to a continuous sub-harmonic function $g_{c, a}(z)$ that is called the Green function of $P_{c, a}$.

We shall write $g_{0}(c, a):=g_{c, a}\left(c_{0}\right), g_{1}(c, a):=g_{c, a}\left(c_{1}\right)$, and

$$
G(c, a):=\max \left\{g_{0}(c, a), g_{1}(c, a)\right\} .
$$

Proposition 2.1. - The function $G(c, a)$ extends continuously to the analytification $\mathbb{A}_{K}^{2, \text { an }}$, and there exists a constant $C=C(K)>0$ such that

$$
\sup _{\mathbb{A}_{K}^{2, \text { an }}}\left|G(c, a)-\log ^{+} \max \{|a|,|c|\}\right| \leq C,
$$

and this constant vanishes when the residual characteristic of $K$ is at least 5 .

Proof. - A proof of this fact is given in [BH2, §4] (see also [DF, §6] for a more detailed proof) in the Archimedean case. When the normed field is non-Archimedean, it is proved in [FG, Proposition 2.5] that the sequence $h_{n}:=\max \left\{\frac{1}{3^{n}} \log ^{+}\left|P_{c, a}^{n}\left(c_{0}\right)\right|, \frac{1}{3^{n}} \log ^{+}\left|P_{c, a}^{n}\left(c_{1}\right)\right|\right\}$ converges uniformly on bounded sets in $K^{2}$ to $G(c, a)$. Since $h_{n}$ extends continuously to $\mathbb{A}_{K}^{2, \text { an }}$, it follows that $G$ too. The rest of the proposition also follows from op. cit.

\subsection{Expansion of the Böttcher coordinate}

For any cubic polynomial $P \in K[z]$, we let the Böttcher coordinate of $P$ be the only formal power series $\varphi$ satisfying the equation

$$
\varphi \circ P(z)=\varphi(z)^{3}
$$

which is of the form

$$
\varphi(z)=\omega z+\alpha+\sum_{k \geq 1} a_{k} z^{-k}
$$

with $\alpha, a_{k} \in K$ for all $k \geq 1$.

Lemma 2.2. - Given any $(c, a) \in K \times K$, the Böttcher coordinate $\varphi_{c, a}(z)$ of the cubic polynomial $P_{c, a}:=\frac{z^{3}}{3}-\frac{c}{2} z^{2}+a^{3}$ exists, is unique, and satisfies

$$
\varphi_{c, a}(z)=\omega\left(z-\frac{c}{2}\right)+\sum_{k \geq 1} a_{k}(c, a) z^{-k}
$$


where

$$
a_{k}(c, a) \in \mathbb{Z}\left[\omega, \frac{1}{2}\right][c, a] \text { with } \operatorname{deg}\left(a_{k}\right)=k+1
$$

Moreover the 2-adic (resp. 3-adic) norm of the coefficients of $a_{k}$ are bounded from above by $2^{k+1}$ (resp. $\left.3^{k / 2}\right)$.

Proof. — The defining equation (2) reads as follows:

$$
\begin{aligned}
\left(\omega\left(z-\frac{c}{2}\right)+\sum_{k \geq 1} a_{k}(c, a) z^{-k}\right)^{3}= & \\
& \omega\left(\frac{z^{3}}{3}-\frac{c}{2} z^{2}+a^{3}-\frac{c}{2}\right)+\sum_{k \geq 1} \frac{3^{k} a_{k}(c, a)}{z^{3 k}\left(1-\frac{3 c}{2 z}+\frac{3 a^{3}}{z^{3}}\right)^{k}}
\end{aligned}
$$

An immediate check shows that terms in $z^{3}$ and $z^{2}$ are identical on both sides of the equation. Identifying terms in $z$ yields

$$
3 \omega^{3}\left(c^{2} / 4\right)+3 \omega^{2} a_{1}=0, \text { so that } a_{1}=-\frac{\omega}{4} c^{2},
$$

whereas identifying constant terms, we get

$$
3 \omega^{2} a_{2}+6 \omega^{2}(-c / 2) a_{1}+\omega^{3}\left(-c^{3} / 8\right)=\omega\left(a^{3}-c / 2\right)
$$

hence

$$
a_{2}=-\frac{5 \omega}{24} c^{3}+\frac{1}{3 \omega}\left(a^{3}-\frac{c}{2}\right)
$$

This shows (4) for $k=1,2$, since $\omega^{-1}=3 \omega$.

We now proceed by induction. Suppose (4) has been proven for $k$. Identifying terms in $z^{-(k-1)}$ in the equation above, we get

$$
\begin{gathered}
3 \omega^{2} a_{k+1}-3 c \omega^{2} a_{k}+\frac{3 \omega^{2}}{4} c^{2} a_{k-1}+ \\
+\omega \sum_{i+j=k} a_{i} a_{j}-\omega \frac{c}{2} \sum_{i+j=k-1} a_{i} a_{j}+\sum_{i+j+l=k+1} a_{i} a_{j} a_{l}= \\
\sum_{l \geq 1} 3^{l} a_{l}\left[\left(1+\frac{3 c}{2 z}+\frac{a^{3}}{z^{3}}\right)^{-l}\right]_{k+1-3 l}
\end{gathered}
$$

where $\left[\left(1+\frac{3 c}{2 z}+\frac{a^{3}}{z^{3}}\right)^{-l}\right]_{j}$ denotes the coefficient in $z^{-j}$ of the expansion of $\left(1+\frac{3 c}{2 z}+\frac{a^{3}}{z^{3}}\right)^{-l}$ in power of $z^{-1}$. Observe that this coefficient belongs to $\mathbb{Z}\left[\frac{1}{2}\right][c, a]$, has 2-adic norm $\leq 2^{l}$, and is a polynomial in $c, a$ of degree at most $j$. It follows that the polynomial

$$
a_{l}(c, a)\left[\left(1+\frac{3 c}{2 z}+\frac{a^{3}}{z^{3}}\right)^{-l}\right]_{k+1-3 l}
$$

is of degree at most $k+1-3 l+l+1=k+2-2 l<k+1$. The induction step is then easy to complete using again $\omega^{-1}=3 \omega$. 


\subsection{Extending the Böttcher coordinate}

Recall that $G(c, a)=\max \left\{g_{0}(c, a), g_{1}(c, a)\right\}$.

Proposition 2.3. - There exists a constant $\rho=\rho(K) \geq 0$ such that the Böttcher coordinate of $P_{c, a}$ is converging in $\{z, \log |z|>\rho+G(c, a)\}$.

There exists another constant $\tau=\tau(K) \geq 0$ such that the map $(c, a, z) \mapsto \varphi_{c, a}(z)$ extends as an analytic map on the open set

$$
\left\{(c, a, z) \in \mathbb{A}_{K}^{2, \text { an }} \times \mathbb{A}_{K}^{1, \text { an }}, g_{c, a}(z)>G(c, a)+\tau\right\},
$$

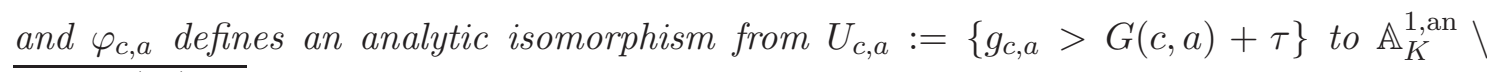
$\overline{\mathbb{D}\left(0, e^{G(c, a)+\tau}\right)}$ satisfying the equation (2) on $U_{c, a}$. We have

$$
g_{c, a}(z)=\log \left|\varphi_{c, a}(z)\right|_{K} \quad \text { on } U_{c, a} .
$$

Finally, $\tau=0$ except if the residual characteristic of $K$ is equal to 2 or 3.

We shall use the following lemma which follows easily from e.g. [FG, Proposition 2.3].

Lemma 2.4. - There exists a constant $\theta=\theta(K) \geq 0$

$$
\sup _{\mathbb{A}_{K}^{1, a n}}\left|g_{c, a}(z)-\log ^{+}\right| z|| \leq \theta .
$$

Moreover, $\theta$ is equal to 0 except if the norm on $K$ is Archimedean or the residual characteristic of $K$ is equal to 2 or 3.

Proof of Proposition 2.3. - Assume first that $K$ is Archimedean, and set $\tau=0$. In that case most of the statements are proved in [DH] (see also [BH2, §1]). In particular, $\varphi_{c, a}(z)$ is analytic in a neighborhood of $\infty$ and extends to $U_{c, a}$ by invariance and defines an isomorphism between the claimed domains. It is moreover analytic in $c, a, z$.

To estimate more precisely the radius of convergence of the power series (31), we rely on [BH2, $\S 4]$ as formulated in [DF, $\S 6]$. First choose $C=C_{K}>0$ such that $G(c, a)>$ $\log ^{+} \max \{|a|,|c|\}-C$. Then $\log |z|>C+G(c, a)$ implies $\left|z-\frac{c}{2}\right|>\max \{1,|a|,|c|\}-\left|\frac{c}{2}\right| \geq$ $\frac{1}{2} \max \{1,|a|,|c|\}$ hence $\log \left|z-\frac{c}{2}\right|>G(c, a)-\log 2$, so that $g_{c, a}(z)>\log \left|z-\frac{c}{2}\right|-\log 4>$ $G(c, a)$, and $\varphi$ converges in $\{z, \log |z|>G(c, a)+\rho\}$ with $\rho:=C$ as required.

From now on, we assume that the norm on $K$ is non-Archimedean.

When the residual characteristic of $K$ is different from 2 and 3, then (4) implies $\left|a_{k}\right| \leq$ $\max \{1,|c|,|a|\}^{k+1}$ so that $\varphi$ converges for $|z|>\max \{1,|c|,|a|\}$, and $\log |\varphi(z)|=\log |z|$. Recall that we have $G(c, a)=\log \max \{1,|c|,|a|\}$ by Proposition 2.1 so that one can take $\rho=0$. Pick any $z$ such that $g(z)>G(c, a)$, and observe that $\left|P^{n}(z)\right| \rightarrow \infty$. Then we get

$$
g_{c, a}(z)=\lim _{n \rightarrow \infty} \frac{1}{3^{n}} \log \left|P^{n}(z)\right|=\lim _{n \rightarrow \infty} \frac{1}{3^{n}} \log \left|\varphi\left(P^{n}(z)\right)\right|=\log |\varphi(z)|=\log |z| .
$$

In particular the set $\{g>G(c, a)\}$ is equal to $\mathbb{A}_{K}^{1, \text { an }} \backslash \overline{\mathbb{D}\left(0, e^{G(c, a)}\right)}$, and $\varphi$ is an analytic map from that open set onto itself. It is an isomorphism since $\log |\varphi(z)|=\log |z|$ as soon as $g(z)>G(c, a)$. The proposition is thus proved in this case with $\tau=0$.

In residual characteristic $2,\left|a_{k}\right| \leq(2 \max \{1,|c|,|a|\})^{k+1}$ whence $\varphi$ converges for $|z|>$ $2 \max \{1,|c|,|a|\}$, and as above $\log |\varphi|=\log |z|$ in that range. Recall that $G(c, a)-$ $\log ^{+} \max \{|c|,|a|\} \geq C=C(K)$, so that $\log |z|>G(c, a)+\log 2-C_{K}$ implies $|z|>$ $2 \max \{1,|c|,|a|\}$, which proves that the power series (3) converges for $\log |z|>G(c, a)+\rho$ 
with $\rho=\log 2-C$. Set $\tau:=\rho+\theta$ where $\theta$ is the constant given by Lemma 2.4. Using $\log |\varphi(z)|=\log |z|$ as above, we get that $\varphi_{c, a}$ defines an analytic isomorphism from $U_{c, a}:=\left\{g_{c, a}>G(c, a)+\tau\right\}$ to $\mathbb{A}_{K}^{1, \text { an }} \backslash \overline{\mathbb{D}\left(0, e^{G(c, a)+\tau}\right)}$.

In residual characteristic $3,\left|a_{k}\right| \leq\left(3^{1 / 2} \max \{1,|c|,|a|\}\right)^{k+1}$ whence $\varphi$ converges $|z|>3^{1 / 2} \max \{1,|c|,|a|\}$, and $\log |\varphi|=\log |\omega z|$ in that range. Recall that $G(c, a)-$ $\log ^{+} \max \{|c|,|a|\} \geq C=C(K)$, so that $\log |z|>G(c, a)+\log 3^{1 / 2}-C_{K}$ implies $|z|>3^{1 / 2} \max \{1,|c|,|a|\}$, which proves that the power series (3i) converges for $\log |z|>G(c, a)+\rho$ with $\rho=\log 3^{1 / 2}-C$. We conclude the proof putting $\tau:=\rho+\theta$ as before.

Remark. - It is possible to argue that $\tau=0$ also in residual characteristic 2. Although we do not know the optimal constant $\tau$ in residual characteristic 3, the Böttcher coordinate is likely not to induce an isomorphism from $\left\{g_{c, a}>G(c, a)\right\}$ to $\mathbb{A}_{K}^{1, \text { an }} \backslash \overline{\mathbb{D}\left(0, e^{G(c, a)}\right)}$.

\section{Curves in $\mathrm{Poly}_{3}$}

In this section we fix a number field $\mathbb{K}$ containing a square-root $\omega$ of $\frac{1}{3}$ and take an irreducible curve $C$ in Poly $_{3}$ that is defined over $\mathbb{K}$. Our aim is to build suitable height functions on $C$ for which the distribution of points of small height can be described using Thuillier-Yuan's theorem. Our main statement is Theorem 3.12 below.

Recall that given any finite set $S$ of places of $\mathbb{K}$ containing all Archimedean places, $\mathcal{O}_{\mathbb{K}, S}$ denotes the ring of $S$-integers in $\mathbb{K}$ that is of elements of $\mathbb{K}$ of $v$-norm $\leq 1$ for all $v \notin S$. We also write $\mathbb{K}_{v}$ for the completion of $\mathbb{K}$ w.r.t. the $v$-adic norm.

\subsection{Adelic series}

A formal power series $\sum_{n} a_{n} z^{n}$ is said to be adelic on $\mathbb{K}$ if its coefficients belong to $\mathcal{O}_{\mathbb{K}, S}$ where $\mathbb{K}$ is a number field, and $S$ a finite set of places on $\mathbb{K}$; and for each place $v$ on $\mathbb{K}$ the series has a positive radius of convergence $r_{v}:=\lim _{\sup _{n \rightarrow \infty}}\left|a_{n}\right|_{v}^{-1 / n}>0$. Observe that $r_{v}=1$ for all but finitely many places.

Lemma 3.1. - Suppose $\alpha(t)=\sum_{n} a_{n} t^{n}$ is an adelic series with $a_{0}=0$ and $a_{1} \neq 0$. Then there exists an adelic series $\beta$ such that $\beta \circ \alpha(t)=t$.

Proof. - Suppose $a_{n} \in \mathcal{O}_{\mathbb{K}, S}$ for all $n$, and write $\beta(t)=\sum_{n} b_{n} t^{n}$. The equation $\beta \circ \alpha(t)=t$ amounts to $b_{0}=0, b_{1}=a_{1}^{-1}$, and the relations

$$
b_{n} a_{1}^{n}+\sum_{1 \leq k \leq n-1} b_{k}\left[\left(\sum_{j \leq n} a_{j} t^{j}\right)^{k}\right]_{n}=0,
$$

for any $n \geq 2$ where $[\cdot]_{n}$ denotes the coefficient in $t^{n}$ of the power series inside the brackets. It follows that $b_{n} \in \mathcal{O}_{\mathbb{K}, S^{\prime}}$ for all $n$ where $S^{\prime}$ is the union of $S$ and all places $v$ for which $\left|a_{1}\right|_{v}>1$. The convergence of the series follows from Cauchy-Kowalewskaia's method of majorant series or from the analytic implicit function theorem, see $[\mathbf{C}$ and $[\mathbf{S e}$, p. 73].

Lemma 3.2. - Pick $k \in \mathbb{N}^{*}$, and suppose $\alpha(t)=\sum_{n \geq k} a_{n} t^{n}$ is an adelic series with $a_{k} \neq 0$. Then there exists an adelic series $\beta$ such that $\beta(t)^{k}=\alpha(t)$. 
Proof. - As in the previous proof, suppose $a_{n} \in \mathcal{O}_{\mathbb{K}, S}$ for all $n$, and write $\beta(t)=b_{1} t+$ $\sum_{n \geq 2} b_{n} t^{n}$. We get $b_{1}^{k}=a_{1}$, and for all $n \geq 2$

$$
a_{n}=k b_{1}^{k-1} b_{n-k}+P_{n}\left(b_{1}, \ldots, b_{n-k-1}\right),
$$

where $P_{n}$ is a polynomial with integral coefficients. This time all coefficients $b_{n}$ belong to a finite extension of $\mathbb{K}$ containing a fixed $k$-th root of $a_{1}$, and $S^{\prime}$ is the union of $S$ and all places $v$ such that $\left|k b_{1}^{k-1}\right|_{v}<1$. The analyticity of the series is handled as in the previous proof.

Lemma 3.3. - Pick $k \in \mathbb{N}^{*}$, and suppose $\alpha(t)=\sum_{n \geq k} a_{n} t^{n}$ is an adelic series with $a_{k} \neq 0$. Then there exists an adelic series $\beta$ such that $\alpha \circ \beta(t)=t^{k}$.

Proof. - The equation $\alpha \circ \beta(t)=t^{k}$ is equivalent to

$$
\left(1+\sum_{j \geq 2} a_{j} t^{j-1}\right)^{k}\left(1+\sum_{l \geq 1} \alpha_{l}\left(t+\sum_{i \geq 2} a_{i} t^{i}\right)^{l}\right)=1 .
$$

Identifying terms of order $t^{n}$, one obtains

$$
k a_{n+1}+\left[\left(1+\sum_{2 \leq j \leq n} a_{j} t^{j-1}\right)^{k}\left(1+\sum_{l \geq 1} \alpha_{l}\left(t+\sum_{1 \leq i \leq n} a_{i} t^{i}\right)\right)\right]_{n}=0
$$

which shows that $\beta$ is unique, has coefficients in $\mathcal{O}_{\mathbb{L}, S^{\prime}}$ where $S^{\prime}$ contains $S$ and all places at which $|k|_{v}<1$. The fact that $\beta$ is analytic at all places is a consequence of the inverse function theorem and the fact that the power series $t \mapsto(1+t)^{1 / n}:=1+\frac{1}{n} t+$ $\frac{(1 / n)(1 / n-1)}{2} t^{2}+O\left(t^{3}\right)$ has a positive radius of convergence.

We shall also deal with adelic series at infinity which we define to be series of the form $\alpha(z)=\sum_{0 \leq k \leq N} b_{k} z^{k}+\sum_{k \geq 1} \frac{a_{k}}{z^{k}}$ with $N \in \mathbb{N}, b_{k}, a_{k} \in \mathcal{O}_{\mathbb{K}, S}$ and $\sum_{k \geq 1} a_{k} t^{k}$ is an adelic series. Observe that this is equivalent to assume that $\alpha\left(t^{-1}\right)^{-1}$ is an adelic series.

\subsection{Puiseux expansions}

We shall need the following facts on the Puiseux parameterizations of a curve defined over $\mathbb{K}$. These are probably well-known but we include a proof for the convenience of the reader.

Proposition 3.4. - Suppose $P \in \mathbb{K}[x, y]$ is a polynomial such that $P(0,0)=0$ and $P(0, y)$ is not identically zero. Denote by $\mathrm{n}: \hat{D} \rightarrow D:=\{P=0\}$ the normalization map, and pick any point $\mathfrak{c} \in \mathrm{n}^{-1}(0) \in \hat{D}$.

Then one can find a finite extension $\mathbb{L}$ of $\mathbb{K}$, a finite set of places $S$ of $\mathbb{L}$, a positive integer $n>0$, and an adelic series $\beta(t) \in \mathcal{O}_{\mathbb{L}, S}[[t]]$ such that

1. there is an isomorphism of complete local rings $\widehat{\mathcal{O}_{\hat{D}, \mathrm{c}}} \simeq \mathbb{L}[[t]]$;

2. the formal map $t \mapsto\left(t^{n}, \beta(t)\right)$ parameterizes the branch $\mathfrak{c}$ in the sense that $x(\mathrm{n}(t))=$ $t^{n}$, and $y(\mathrm{n}(t))=\beta(t)$.

A branch of $D$ at the origin is by definition a point in $\mathrm{n}^{-1}(0)$. 
Proof. - We first reduce the situation to the case $D$ is smooth at 0 . To do so we blow-up the origin $X_{1} \rightarrow \mathbb{A}^{2}$ and let $D_{1}$ be the strict transform of $D$. Since $\hat{D}$ is normal the map $\mathrm{n}$ lifts to a map $\mathrm{n}_{1}: \hat{D} \rightarrow D_{1}$, and we let $p_{1}$ be the image of $\mathfrak{c}$ in $D_{1}$.

In the coordinates $(x, y)=\left(x^{\prime}, x^{\prime} y^{\prime}\right)$ (or $\left.\left(x^{\prime} y^{\prime}, y^{\prime}\right)\right)$ the point $p_{1}$ has coordinates $\left(0, y_{1}\right)$ where $y_{1}$ is the solution of a polynomial with values in $\mathbb{K}$ hence belongs to an algebraic extension of this field. We may thus choose charts $(x, y)=\left(x^{\prime},\left(x^{\prime}+c\right) y^{\prime}\right)\left(\right.$ or $\left.\left(x^{\prime}\left(y^{\prime}+c\right), x^{\prime}\right)\right)$ with $c \in \overline{\mathbb{K}}$ such that $\mathfrak{c}$ is now a branch of $D_{1}=\left\{P_{1}=0\right\}$ at the origin, and $P_{1} \in \overline{\mathbb{K}}\left[x^{\prime}, y^{\prime}\right]$.

We iterate this process of blowing-up to build a sequence of proper birational morphisms between smooth surfaces $X_{i+1} \rightarrow X_{i}, i=1, \ldots, N$ until we arrive at the following situation for $X:=X_{N}$ : the strict transform $C$ of $D$ by $\pi: X \rightarrow \mathbb{A}^{2}$ is smooth at a point $p \in \pi^{-1}(0)$ and intersects transversally the exceptional locus of $\pi$.

The normalization map $\mathrm{n}: \hat{D} \rightarrow D$ lifts to a map $\mathrm{m}: \hat{D} \rightarrow C$ and the image of $\mathfrak{c}$ by $\mathrm{m}$ is equal to $p$. Finally there exist coordinates $z, w$ centered at $p$ such that $(x, y)=\pi(z, w)=$ $(A(z, w), B(z, w))$ with $A, B \in \overline{\mathbb{K}}[z, w]$, the exceptional locus of $\pi$ contains $\{z=0\}$, and $C=\{R(z, w):=w-z a(z)-w Q(z, w)=0\}$ where $a \in \overline{\mathbb{K}}[z], Q \in \overline{\mathbb{K}}[z, w]$ and $Q(0,0)=0$.

Fix an algebraic extension $\mathbb{L}$ of $\mathbb{K}$ and $S$ finitely many places of $\mathbb{L}$ such that $A, B, R$ have their coefficients in $\mathcal{O}_{\mathbb{L}, S}$.

We now look for a power series $\gamma(t)=\sum_{k>1} \gamma_{k} t^{k}$ such that $R(t, \gamma(t))=0$. Its coefficients satisfy the relations

$$
\gamma_{k}=\left[t^{2} a(t)\right]_{k}+\left[\left(\sum_{j=1}^{k-1} \gamma_{j} t^{j}\right) Q\left(t, \sum_{j=1}^{k-1} \gamma_{j} t^{j}\right)\right]_{k}
$$

which implies that $\gamma$ exists, is unique, and all its coefficients belongs to $\mathcal{O}_{\mathbb{L}, S}$. It follows from the analytic implicit function theorem, that $\gamma$ is also analytic as a power series in $\mathbb{L}_{v}[[t]]$ for any place $v$.

Let us now consider the two power series $(\alpha(t), \delta(t)):=\pi(t, \gamma(t))$. They both belong to $\mathcal{O}_{\mathbb{L}, S}$, are analytic at any place, and we have $P(\alpha(t), \delta(t))=0$. Since $P(0, y)$ is not identically zero, we may write $\alpha(t)=t^{n}\left(a+\sum_{k>1} \alpha_{k} t^{k}\right)$ for some $n>0$ and $a \neq 0$. Replacing $\mathbb{L}$ by a suitable finite extension, and $t$ by $a^{\prime} t$ for a suitable $a^{\prime}$ we may suppose that $a=1$ and $\alpha_{k} \in \mathcal{O}_{\mathbb{L}, S}$ for all $k$.

By Lemma 3.3. there exists an invertible power series $\hat{a}(t)=t+\sum_{k \geq 2} a_{k} t^{k}$ that is analytic at all places with coefficients $a_{k} \in \mathcal{O}_{\mathbb{L}, S}$ and such that $\alpha \circ \hat{a}(t)=t^{n}$. Once this claim is proved one sets $\beta(t):=\delta \circ \hat{a}(t)$, so that $\pi(\hat{a}(t), \gamma(\hat{a}(t)))=\left(t^{n}, \beta(t)\right)$.

Since $\mathrm{m}$ is injective and maps the smooth point $\mathfrak{c} \in \hat{D}$ to the smooth point $p \in C$, it induces an isomorphism of complete local rings $\widehat{\mathcal{O}_{C, p}} \simeq \widehat{\mathcal{O}_{\hat{D}, c}}$. Observe that the complete local ring $\widehat{\mathcal{O}_{C, p}}=\mathbb{L}[[z, w]] /\langle R\rangle$ is isomorphic to $\mathbb{L}[[t]]$ by sending the class of a formal series $\Phi$ to $\Phi(t, \gamma(t)))$. Composing with the isomorphism of $\mathbb{L}[[t]]$ sending $t$ to $\hat{a}(t)$, we get an isomorphism $\widehat{\mathcal{O}_{\hat{D}, \mathfrak{c}}} \simeq \mathbb{L}[[t]]$ such that $(x(\mathrm{n}(t)), y(\mathrm{n}(t)))=\pi(\mathrm{n}(t))=\pi(\hat{a}(t), \gamma(\hat{a}(t)))=$ $\left(t^{n}, \beta(t)\right)$ as required.

\subsection{Branches at infinity of a curve in Poly $_{3}$}

Consider an irreducible affine curve $C \subset$ Poly $_{3}$ defined over a number field $\mathbb{K}$. We denote by $\overline{\text { Poly }_{3}} \simeq \mathbb{P}^{2}$ the natural compactification of Poly ${ }_{3} \simeq \mathbb{A}^{2}$ using the affine coordinates $(c, a)$. Let $\bar{C}$ be the Zariski closure of the curve $C$ in $\overline{\text { Poly }_{3}}$, and $\mathrm{n}: \hat{C} \rightarrow \bar{C}$ be its normalization. A branch at infinity of $C$ is a point in $\hat{C}$ lying over $\bar{C} \backslash C$. 
Proposition 3.5. - There exists a finite extension $\mathbb{L}$ of $\mathbb{K}$ and a finite set of places $S$ such that the following holds.

For any branch $\mathfrak{c}$ of $C$ at infinity there is an isomorphism of complete local rings $\widehat{\mathcal{O}_{\hat{C}, \mathfrak{c}}} \simeq$ $\mathbb{L}[[t]]$ such that $c(\mathrm{n}(t)), a(\mathrm{n}(t))$ are adelic series at infinity.

Proof. - Pick a branch at infinity $\mathfrak{c}$ of $C$. Let $p_{*}$ be the image of $\mathfrak{c}$ in $\overline{\text { Poly }_{3}} \simeq \mathbb{P}^{2}$. It is given in homogeneous coordinates by $p_{*}=\left[c_{*}: a_{*}: 0\right]$ and since $C$ is defined over $\mathbb{K}$ we may assume $c_{*}, a_{*}$ are algebraic over $\mathbb{K}$. To simplify the discussion we shall assume that $c_{*}=1$ so that $p_{*}=\left[1: a_{*}: 0\right]$ (otherwise $p_{*}=[0: 1: 0]$ and the arguments are completely analoguous). Let $d$ be the degree of a defining equation $P \in \mathbb{K}[c, a]$ of $C$. Observe that $Q(\tau, \alpha):=\tau^{d} P\left(\frac{1}{\tau}, \frac{\alpha}{\tau}-a_{*}\right)$ is a polynomial vanishing at $(0,0)$ such that $Q(0, \alpha)$ is not identically zero. Note that $\{Q=0\}$ can be identified to an open Zariski subset of the completion of $\{P=0\}$ in $\overline{\text { Poly }_{3}}$, and $\mathfrak{c}$ with a branch of $\{Q=0\}$ at the origin.

Apply Proposition 3.4 to this branch $\mathfrak{c}$. We get a finite extension $\mathbb{L}$, a finite set of places $S$ of $\mathbb{L}$ containing all archimedean ones, a positive integer $n$, an isomorphism of complete local ring $\mathcal{O}_{\hat{C}, \mathfrak{c}} \simeq \mathbb{L}[[t]]$, and a power series $\beta \in \mathcal{O}_{\mathbb{L}, S}[[t]]$ that is analytic at all places such that $\alpha(\mathrm{n}(t))=\beta(t)$ and $\tau(\mathrm{n}(t))=t^{n}$. It follows that $c(\mathrm{n}(t))=t^{-n}$, and $a(\mathrm{n}(t))=t^{-n} \beta(t)-a_{*} \in \mathcal{O}_{\mathbb{L}, S}[[t]]$.

\subsection{Estimates for the Green functions on a curve in $\mathbf{P o l y}_{3}$}

In this section, we fix an irreducible curve $C$ in Poly $_{3}$ defined over a number field $\mathbb{K}$ and let $\mathbb{L}$ be a finite extension of $\mathbb{K}$ for which Proposition 3.5 applies. Fix a place $v$ of $\mathbb{L}$, and let $g_{0, v}(c, a)$ be the function $g_{0, v}$ evaluated at $c, a$ in the completion $\mathbb{L}_{v}$ of $\mathbb{L}$ with respect to the $v$-adic norm.

By [DF] and [FG, Proposition 2.4], the function $g_{0, v}$ is the uniform limit on compact sets of $\frac{1}{3^{n}} \log ^{+}\left|P_{c, a}^{n}\left(c_{0}\right)\right|_{v}$. It follows that its lift to the normalization of $C$ is sub-harmonic (in the classical sense when $v$ is Archimedean and in the sense of Thuillier [T] when $v$ is non-Archimedean).

To simplify notations, we also write $g_{0, v}(t):=g_{0, v}(c(\mathrm{n}(t)), a(\mathrm{n}(t)))$ where the adelic series at infinity $c(\mathrm{n}(t))$ and $a(\mathrm{n}(t))$ are given as above.

Proposition 3.6. - For each branch $\mathfrak{c}$ of $C$ at infinity, one of the following two situations occur.

1. For any place $v$ of $\mathbb{L}$, the function $g_{0, v}(t)$ extends as a locally bounded subharmonic function through $\mathfrak{c}$.

2. There exist a finite set of place $S$ of $\mathbb{L}$, and two constants $a(\mathfrak{c}) \in \mathbb{Q}_{+}^{*}$ and $b(\mathfrak{c}) \in \mathcal{O}_{\mathbb{L}, S}$ such that $g_{0, v}(t)=a(\mathfrak{c}) \log |t|_{v}^{-1}+\log |b(\mathfrak{c})|_{v}+o(1)$ for any place $v$ on $\mathbb{L}$.

Remark. - This key result is very similar to [BDM, Proposition 2.1]. Ghioca and Ye have proved that $g_{0, v}(t)$ actually extends to a continuous function at $t=0$ in case 1 . We also refer to [De2, Proposition 3.1] for a version of this result in the case of rational maps.

Notation. - We endow the field $\mathbb{L}((t))$ with the $t$-adic norm so that for any Laurent series $Q=\sum a_{k} t^{k}$ we have $|Q|_{t}:=e^{-\operatorname{ord}_{t}(Q)}$ with $\operatorname{ord}_{t}(Q)=\min \left\{k, a_{k} \neq 0\right\}$. The resulting valued field is complete and non-Archimedean.

In order to avoid confusion, we denote by $\mathrm{P}(z) \in \mathbb{L}((t))[z]$ the cubic polynomial induced by the family $\left(P_{c(\mathrm{n}(t)), a(\mathrm{n}(t))}\right)_{t}$. Observe that the critical points of $\mathrm{P}$ are given by $\mathrm{c}_{0}$ and $\mathrm{c}_{1}$ which correspond to the adelic series at infinity 0 and $c(\mathrm{n}(t))$ respectively. 
Proof of Proposition 3.6. - For each $q \in \mathbb{N}^{*}$, we set $e_{q}:=\left|\mathrm{P}^{q}\left(\mathrm{c}_{0}\right)\right|_{t}$, so that either the sequence $\left\{e_{q}\right\}_{q \in \mathbb{N}}$ is bounded (i.e. $c_{0}$ belongs to the filled-in Julia set of $\mathrm{P}$ ) or $e_{q} \rightarrow \infty$ (exponentially fast).

Suppose we are in the former case, and consider the sequence of subharmonic functions $\frac{1}{3^{q}} \log ^{+}\left|P_{t}^{q}(0)\right|_{v}$ defined on a punctured disk $\mathbb{D}_{v}^{*}$ centered at 0 in $\mathbb{A}_{\mathbb{L}_{v}}^{1, \text { an }}$. Since $\frac{1}{3^{q}} \log ^{+}\left|P_{t}^{q}(0)\right|_{v}=\frac{\log ^{+} e_{q}}{3^{q}} \log |t|_{v}^{-1}+O(1)$, the function

$$
h_{q}:=\frac{1}{3^{q}} \log ^{+}\left|P_{t}^{q}(0)\right|_{v}-\frac{\log ^{+} e_{q}}{3^{q}} \log |t|_{v}^{-1}
$$

is subharmonic on $\mathbb{D}_{v}^{*}$ and locally bounded near 0 . It thus extends as a subharmonic function to $\mathbb{D}_{v}$ by the next lemma.

Lemma 3.7. - Any subharmonic function on $\mathbb{D}_{v}^{*}$ that is bounded from above in a neighborhood of the origin is the restriction of a subharmonic function on $\mathbb{D}_{v}$.

Proof. - In the Archimedean case, this follows from [H2, Theorem 3.4.3]. Let us explain how to adapt these arguments to the non-Archimedean setting. Let $h$ be a subharmonic function on $\mathbb{D}_{v}^{*}$, and suppose it is non-positive. For each integer $n$ consider the function $h_{n}:=h+\frac{1}{n} \log |t|$ with the convention $h_{n}(0)=-\infty$. Observe that $h_{n}$ is the pointwise decreasing limit of the sequence of subharmonic functions $\max \left\{h_{n},-A\right\}$ as $A \rightarrow \infty$, hence is subharmonic by [T Proposition 3.1.9]. Letting $n \rightarrow \infty$, we get an increasing sequence of subharmonic functions that is locally bounded and converging pointwise on $\mathbb{D}_{v}^{*}$ to $h$. The upper-semicontinuous regularization $h^{*}$ of $\lim _{n} h_{n}$ is thus subharmonic on $\mathbb{D}_{v}^{*}$ by $[\mathbf{T}$, Proposition 3.1.9] and extends $h$ as required.

Since $\frac{1}{3^{q}} \log ^{+}\left|P_{t}^{q}(0)\right|_{v}$ converges uniformly on compact subsets in $\mathbb{D}_{v}^{*}$ to $g_{0, v}, h_{q}$ is uniformly bounded from above on its boundary, hence everywhere by the maximum principle. It follows from Hartog's theorem (see e.g. [H1, Theorem 1.6.13] in the Archimedean case, and [FRL, Proposition 2.18] in the non-Archimedean case) that $h_{q}$ converges (in $L_{\text {loc }}^{1}$ in the Archimedean case, and pointwise at any non-rigid point in the non-Archimedean case) to a subharmonic function, hence $g_{0, v}$ is subharmonic on $\mathbb{D}_{v}$. But $g_{0, v}$ is non-negative so that (1) holds.

Suppose that $e_{q} \rightarrow \infty$. Recall that $c(\mathrm{n}(t))$ and $a(\mathrm{n}(t))$ are adelic series at infinity that belong to $t^{-n} \mathcal{O}_{\mathbb{L}, S}[[t]]$ for a suitable integer $n \geq 1$. Write $\varphi_{t}:=\varphi_{\left.P_{c(\mathrm{n}(t)), a(\mathrm{n}(t))}\right)}$.

Lemma 3.8. - There exists an integer $q \geq 1$ such that for any place $v$ of $\mathbb{L}$, there exists $\epsilon>0$ such that $P_{t}^{q}\left(c_{0}\right)$ belongs to the domain of convergence of $\varphi_{t}$ for any $|t|_{v}<\epsilon$.

Proof. - Indeed $P_{t}^{q}\left(c_{0}\right)$ is an adelic series at infinity having a pole of order $\log e_{q}$. On the other hand, we have

$$
\begin{aligned}
G(t):=G(c(\mathrm{n}(t)), a(\mathrm{n}(t))) & \leq \log \max \{|c(\mathrm{n}(t))|,|a(\mathrm{n}(t))|\}+C \\
& \leq n \log |t|^{-1}+O(1)
\end{aligned}
$$

by Proposition 2.1. By assumption we may take $\log e_{q}$ to be as large as we want so that $\log \left|P_{t}^{q}\left(c_{0}\right)\right|_{v}-G(t) \rightarrow \infty$ for any fixed place $v$ when $|t|_{v} \rightarrow 0$. We conclude by Proposition 2.3.

Our objective is to estimate $\varphi_{t}\left(P_{t}^{q}\left(c_{0}\right)\right)$. Recall from Lemma 2.2 that

$$
\varphi_{c, a}(z)=\omega\left(z-\frac{c}{2}\right)+\sum_{k \geq 1} a_{k}(c, a) z^{-k}
$$


with $a_{k} \in \mathbb{Z}\left[\omega, \frac{1}{2}\right][c, a]$ of degree $\leq k+1$. It follows that

$$
\mathrm{a}_{k}:=a_{k}(c(\mathrm{n}(t)), a(\mathrm{n}(t))) \in t^{-n(k+1)} \mathcal{O}_{\mathbb{L}, S}[[t]],
$$

so that one can define

$$
\varphi_{\mathrm{P}}(z):=\varphi_{c(\mathrm{n}(t)), a(\mathrm{n}(t))}(z)=\omega\left(z-\frac{c(\mathrm{n}(t))}{2}\right)+\sum_{k \geq 1} \mathrm{a}_{k} z^{-k}
$$

as an element of the ring $t^{-n} z \mathcal{O}_{\mathbb{L}, S}((t))\left[\left[\left(t^{n} z\right)^{-1}\right]\right]$.

On the other hand, $P_{c, a}^{q}\left(c_{0}\right)$ is a polynomial in $c, a$ of degree $\leq 3^{q}$ with coefficients in $\mathbb{Z}\left[\frac{1}{2}, \frac{1}{3}\right]$ hence, if

$$
\mathrm{c}_{0}:=c_{0}(\mathrm{n}(t)) \text { and } \mathrm{P}^{q}(z):=P_{c(\mathrm{n}(t)), a(\mathrm{n}(t))}^{q}(z),
$$

we have $\mathrm{P}^{q}\left(\mathrm{c}_{0}\right) \in t^{-3^{q} n} \mathcal{O}_{\mathbb{L}, S}[[t]]$, so that

$$
\frac{\mathrm{a}_{k}}{\left(\mathrm{P}^{q}\left(\mathrm{c}_{0}\right)\right)^{k}} \in t^{3^{q} n k-n(k+1)} \mathcal{O}_{\mathbb{L}, S}[[t]] \subset t^{n k} \mathcal{O}_{\mathbb{L}, S}[[t]] .
$$

It follows that $\Theta:=\sum_{k \geq 1} \frac{a_{k}}{\left(\mathrm{P}^{q}\left(\mathrm{c}_{0}\right)\right)^{k}}$ converges as a formal power series and belongs to $t^{n} \mathcal{O}_{\mathbb{L}, S}[[t]]$. Observe that Lemma 3.8 shows that $\Theta$ is convergent at all places hence defines an adelic power series.

Fix a place $v$ of $\mathbb{L}$ and choose $|t|_{v}$ small enough. Then we get

$$
\begin{aligned}
\varphi_{t}\left(P_{t}^{q}\left(c_{0}\right)\right) & =\omega\left(P_{t}^{q}(0)-\frac{c(\mathrm{n}(t))}{2}\right)+\Theta(t) \\
& =\omega\left(P_{t}^{q}(0)-\frac{c(\mathrm{n}(t))}{2}\right)+o(1) .
\end{aligned}
$$

By (8), for $|t|_{v}$ small enough, one obtains

$$
\begin{aligned}
g_{0, v}(t) & =\frac{1}{3^{q}} \log \left|\varphi_{t}\left(P_{t}^{q}(0)\right)\right|_{v} \\
& =\frac{1}{3^{q}} \log \left|\omega\left(P_{t}^{q}(0)-\frac{1}{2 t^{n}}\right)\right|_{v}+o(1) \\
& =\frac{1}{3^{q}} \log \left|\sum_{0 \leq k \leq n_{0}} \frac{b_{k, 0}}{t^{k}}\right|_{v}+o(1)= \\
& =\frac{n_{0}}{3^{q}} \log |t|_{v}^{-1}+\log \left|\sum_{0 \leq k \leq n_{0}} b_{k, 0} t^{n_{0}-k}\right|_{v}+o(1)
\end{aligned}
$$

where $b_{k, 0} \in \mathcal{O}_{\mathbb{L}, S}$, and $b_{n_{0}, 0} \neq 0$. And the proof is complete with $a(\mathfrak{c}):=\frac{n_{0}}{3^{q}}$, and $b(\mathfrak{c})=b_{n_{0}, 0}$.

Proposition 3.9. - Fix any two positive integers $s:=\left(s_{0}, s_{1}\right)$, and for any place $v$ define

$$
g_{s, v}(c, a):=\max \left\{s_{0} g_{0, v}(c, a), s_{1} g_{1, v}(c, a)\right\} .
$$

Then there exists an integer $q \geq 1$ such that

$$
g_{s, v}(c, a)=\frac{1}{3^{q}} \max \left\{s_{0} \log ^{+}\left|P_{c, a}^{q}\left(c_{0}\right)\right|, s_{1} \log ^{+}\left|P_{c, a}^{q}\left(c_{1}\right)\right|\right\}
$$

for all but finitely many places. 
Proof. - During the proof $S$ is a finite set of places on $\mathbb{L}$ that contains all Archimedean places and all places of residual characteristic 2 and 3. Pick any $v \notin S$, and recall from [FG, Proposition 2.5] that $G_{v}(c, a)=\log ^{+} \max \left\{|c|_{v},|a|_{v}\right\}$.

Suppose first that $g_{s, v}(c, a)=0$. Then $g_{c, a, v}\left(c_{0}\right)=g_{c, a, v}\left(c_{1}\right)=0$ and $G_{v}(c, a)=0$ so that $\frac{1}{3^{q}} \log ^{+}\left|P_{c, a}^{q}\left(c_{0}\right)\right|_{v}=\frac{1}{3^{q}} \log ^{+}\left|P_{c, a}^{q}\left(c_{1}\right)\right|_{v}=0$ for all $q$, and (9) holds in that case.

Pick $q$ large enough such that $3^{q}>\max \left\{\frac{s_{1}}{s_{0}}, \frac{s_{0}}{s_{1}}\right\}$. Suppose now that $0<g_{s, v}(c, a)=$ $s_{0} g_{0, v}(c, a)$ so that $s_{0} g_{0, v}(c, a) \geq s_{1} g_{1, v}(c, a)$. Then

$$
\begin{aligned}
g_{c, a, v}\left(P_{c, a}^{q}\left(c_{0}\right)\right)=3^{q} g_{0, v}(c, a) \geq & \\
& \geq 3^{q} \min \left\{\frac{s_{1}}{s_{0}}, 1\right\} \max \left\{g_{0, v}(c, a), g_{1, v}(c, a)\right\}>G_{v}(c, a) .
\end{aligned}
$$

By (6), we get

$$
g_{s, v}(c, a)=s_{0} g_{0, v}(c, a)=\frac{s_{0}}{3^{q}} g_{0, v}\left(P_{c, a}^{q}\left(c_{0}\right)\right)=\frac{s_{0}}{3^{q}} \log ^{+}\left|P_{c, a}^{q}\left(c_{0}\right)\right|_{v} .
$$

Now observe that either $P_{c, a}^{q}\left(c_{1}\right)$ falls into the domain of definition of $\varphi_{c, a}$ i.e. $\log \left|P_{c, a}^{q}\left(c_{1}\right)\right|_{v}>G_{v}(c, a)$ and $g_{1, v}(c, a)=\frac{1}{3 q} \log ^{+}\left|P_{c, a}^{q}\left(c_{1}\right)\right|_{v}$, so that

$$
\begin{aligned}
g_{s, v}(c, a) & =\max \left\{s_{0} g_{0, v}(c, a), s_{1} g_{1, v}(c, a)\right\} \\
& =\frac{1}{3^{q}} \max \left\{s_{0} \log ^{+}\left|P_{c, a}^{q}\left(c_{0}\right)\right|_{v}, s_{1} \log ^{+}\left|P_{c, a}^{q}\left(c_{1}\right)\right|_{v}\right\},
\end{aligned}
$$

as required. Or we have

$$
\frac{s_{1}}{3^{q}} \log ^{+}\left|P_{c, a}^{q}\left(c_{1}\right)\right|_{v} \leq \frac{s_{1}}{3^{q}} \log ^{+} \max \left\{|a|_{v},|c|_{v}\right\} \leq s_{0} g_{0, v}(c, a),
$$

and again (10) holds.

We complete the proof by arguing in the same way when $g_{s, v}(c, a)=s_{1} g_{1, v}(c, a)$.

\subsection{Adelic semi-positive metrics on curves in Poly ${ }_{3}$}

We fix a number field $\mathbb{L}$ and a finite set $S$ of places of this field that contains all Archimedean places and all places of residual characteristic 2 and 3. We also assume that Propositions 3.5, 3.6 and 3.9 are all valid for these choices.

Fix any pair of positive integers $s_{0}, s_{1} \in \mathbb{N}^{*}$. For each place $v$, introduce the function

$$
g_{s, v}(c, a):=\max \left\{s_{0} \cdot g_{0, v}(c, a), s_{1} \cdot g_{1, v}(c, a)\right\},
$$

as in the previous section.

Pick a branch at infinity $\mathfrak{c}$ and choose parameterizations such that Proposition 3.6 is valid for $g_{0, v}$ and $g_{1, v}$. Observe that

$$
G_{v}(t)=\max \left\{g_{0, v}(t), g_{1, v}(t)\right\} \rightarrow \infty
$$

as $t \rightarrow 0$ by Proposition 2.1 so that either $g_{0, v}$ or $g_{1, v}$ tends to infinity near $t=0$. Since $n_{0}$ and $n_{1}$ are both positive, we conclude to the existence of $a(\mathfrak{c}) \in \mathbb{Q}_{+}^{*}$ and $b(\mathfrak{c}) \in \mathcal{O}_{\mathbb{L}, S}$ such that

$$
g_{s, v}(t)=a(\mathfrak{c}) \log |t|_{v}^{-1}+\log |b(\mathfrak{c})|_{v}+o(1) .
$$

We replace the integers $s_{0}, s_{1}$ by suitable multiples such that the constants $a(\mathfrak{c})$ become integral (for all branch $\mathfrak{c}$ ), and we introduce the divisor $\mathrm{D}:=\sum a(\mathfrak{c})[\mathfrak{c}]$ on $\hat{C}$ where the sum is taken over all branches at infinity of $C$. 
Pick a place $v$, an open subset $U \subset \hat{C}^{\text {an, } v}$ and a section $\sigma$ of the line bundle $\mathcal{O}_{\hat{C}}$ (D) over $U$. By definition $\sigma$ is a meromorphic function on $U$ whose divisor of poles and zeroes satisfies $\operatorname{div}(\sigma)+\mathrm{D} \geq 0$. We set $|\sigma|_{s, v}:=|\sigma|_{v} e^{-g_{s, v}}$.

Recall the notion of semi-positive metrics in the sense of Zhang from [CL2, $\$ 1.2 .8$ \& 1.3.7]. We are now in position to prove

Lemma 3.10. - The metrization $|\cdot|_{s, v}$ on the line bundle $\mathcal{O}_{\hat{C}}(\mathrm{D})$ is continuous and semi-positive for any place $v$. The collection of metrizations $\left\{|\cdot|_{s, v}\right\}_{v}$ is adelic.

Proof. - For any place $v$, and for any local section $\sigma$ of the line bundle $\mathcal{O}_{\hat{C}}(\mathrm{D})$ the function $|\sigma|_{s, v}$ is continuous by (11), therefore the metrization $|\cdot|_{s, v}$ is continuous.

Since $g_{s, v}$ is subharmonic on $C^{v \text {,an }}$, for any local section $\sigma$ the function $-\log |\sigma|_{s, v}$ is subharmonic on $C^{v \text {,an }}$. As it extends continuously to $\hat{C}^{\mathrm{an}, v}$, Lemma 3.7 implies that $-\log |\sigma|_{s, v}$ is subharmonic on $\hat{C}^{\text {an, } v}$. When $v$ is Archimedean, the metrization is thus semi-positive by definition. When $v$ is non-Archimedean the metrization is semi-positive by the next lemma.

Finally the collection of metrizations $\left\{|\cdot|_{s, v}\right\}_{v}$ is adelic thanks to Proposition 3.9 and [FG, §2.3].

Lemma 3.11. - Suppose $L \rightarrow X$ is an ample line bundle on a smooth projective curve over a metrized field. Let $|\cdot|$ be any continuous metrization on $L$ that is subharmonic in the sense that for any local section $\sigma$ the function $-\log |\sigma|$ is subharmonic.

Then the metrization $|\cdot|$ is semi-positive in the sense of Zhang.

Remark. - This result holds true in arbitrary dimension over any field of the form $L((t))$ endowed with the $t$-adic norm where $L$ has characteristic zero by [BFJ].

Proof. - One needs to show that $|\cdot|$ is the uniform limit of a sequence of semi-positive model metrics. By [T Théorème 3.4.15], there exists an inductive set $I$ and an increasing family of semi-positive model metrizations $|\cdot|_{i}$ that are pointwise converging to $|\cdot|$.

Since $|\cdot|$ is continuous and $X$ is compact, Dini's theorem applies and shows that the convergence is uniform.

Observe that the notion of semi-positivity used in op. cit. for model metrics coincides with the one in $[\mathbf{Z}]$ as explained in $[\mathbf{T}, \S 4.3 .2]$.

We have thus obtained

Theorem 3.12. - Pick any positive integers $s_{0}, s_{1}>0$. Then there exists a positive integer $n \geq 1$, and a non-zero effective and integral divisor $\mathrm{D}$ on $\hat{C}$ such that the collection of subharmonic functions

$$
g_{s, v}(c, a):=\max \left\{n s_{0} \cdot g_{0, v}(c, a), n s_{1} \cdot g_{1, v}(c, a)\right\},(c, a) \in C^{v, \text { an }}
$$

induces a semi-positive adelic metrization on the line bundle $\mathcal{O}_{\hat{C}}(\mathrm{D})$.

Remark. - Since the metrization $|\cdot|_{s, v}$ is continuous its curvature form (see [CL2]) does not charge any point, and is given by the pull-back by $\mathrm{n}$ of the positive measure $\Delta g_{s, v}$ restricted to the set of regular points on $C$, see [T, §3.4.3]. To simplify notations we shall simply write this curvature form as $\Delta g_{s, v}$.

Remark. - The line bundle $\mathcal{O}_{\hat{C}}(\mathrm{D})$ is defined over the same number field as $C$. 
Remark. - It is likely that $g_{s, v}$ defines a semi-positive adelic metrization on an ample line bundle over a suitable compactification of $\mathrm{Poly}_{3}$, but this seems quite delicate to prove for arbitrary $s=\left(s_{0}, s_{1}\right) \in\left(\mathbb{N}^{*}\right)^{2}$.

\section{Green functions on special curves}

This section is devoted to the proof of Theorem 4.1 below. If $\mathbb{K}$ is a number field, and $v$ a place of $\mathbb{K}$, recall the definition of $\tau_{v}=\tau\left(\mathbb{K}_{v}\right)$ from Proposition 2.3, and that $\tau_{v}=0$ if the residual characteristic of $\mathbb{K}$ is larger than 5 .

Theorem 4.1. - Let $C$ be an irreducible curve in the space Poly $_{3}$ of complex cubic polynomials parameterized as in (11). Suppose that $C$ contains infinitely many post-critically finite parameters and that neither $c_{0}$ nor $c_{1}$ is persistently pre-periodic. Then the following holds.

1. The curve $C$ is defined over a number field $\mathbb{K}$ and there exist positive integers $s_{0}, s_{1}$ such that for any place $v$ of $\mathbb{K}$

$$
s_{0} g_{0, v}(c, a)=s_{1} g_{1, v}(c, a) \text { for all }(c, a) \in C^{v, \text { an }} .
$$

2. For any branch $\mathfrak{c}$ of $C$ at infinity, there exists an integer $q \geq 1$ and a root of unity $\zeta$ such that for any place $v$ of $\mathbb{K}$, one has

$$
\left(\varphi_{c, a}\left(P_{c, a}^{q}\left(c_{0}\right)\right)\right)^{s_{0}}=\zeta \cdot\left(\varphi_{c, a}\left(P_{c, a}^{q}\left(c_{1}\right)\right)\right)^{s_{1}}
$$

on the connected component of $\left\{g_{0, v}>\tau_{v} / s_{0}\right\}=\left\{g_{1, v}>\tau_{v} / s_{1}\right\}$ in $C^{v \text {,an }}$ whose closure in $\hat{C}$ contains $\mathfrak{c}$.

A remark is in order about the second assertion of the theorem.

Remark. - We shall prove that for any parameter on the connected component $\left\{g_{0, v}>\right.$ $\left.\tau_{v} / s_{0}\right\}=\left\{g_{1, v}>\tau_{v} / s_{1}\right\}$ in $C^{v, \text { an }}$ whose closure in $\hat{C}$ contains $\mathfrak{c}$, the two points $P_{c, a}^{q}\left(c_{0}\right)$ and $P_{c, a}^{q}\left(c_{1}\right)$ belong to the domain of definition of the Böttcher coordinate $\varphi_{c, a}$ for $q$ large so that (12) is consistent. Note that we shall prove that (12) holds as an equality of adelic series at infinity.

\subsection{Green functions are proportional}

The set of post-critically finite polynomials is a countable union of varieties

$$
V_{n, m}:=\left\{P_{c, a}^{n_{0}+m_{0}}\left(c_{0}\right)=P_{c, a}^{n_{0}}\left(c_{0}\right)\right\} \cap\left\{P_{c, a}^{n_{1}+m_{1}}\left(c_{1}\right)=P_{c, a}^{n_{1}}\left(c_{1}\right)\right\}
$$

with $n=\left(n_{0}, n_{1}\right) \in \mathbb{N}^{2}$ and $m=\left(m_{0}, m_{1}\right) \in\left(\mathbb{N}^{*}\right)^{2}$, and each of which is cut out by two polynomial equations with coefficients in $\mathbb{Z}\left[\frac{1}{2}, \frac{1}{3}\right]$. Since $V_{n, m}(\mathbb{C})$ are all contained in a fixed compact set by [BH2] (see also [DF, Proposition 6.2]), it is a finite set, hence all its solutions are defined over a number field.

It follows that $C$ is an irreducible curve containing infinitely many algebraic points $\left(c_{n}, a_{n}\right)$. Let $Q \in \mathbb{C}[c, a]$ be a defining equation for $C$ with at least one coefficient equal to 1 and pick $\sigma$ an element of the Galois group of $\mathbb{C}$ over the algebraic closure of $\mathbb{Q}$. Then $Q \circ \sigma$ vanishes also on $\left\{\left(c_{n}, a_{n}\right)\right\}$ hence everywhere on $C$, and therefore $Q \circ \sigma=\lambda Q$ for some $\lambda \in \mathbb{C}^{*}$. Since one coefficient of $Q$ is 1 , we get $\lambda=1$ and $Q \in \mathbb{K}[c, a]$ for a number field $\mathbb{K}$.

Recall that we denote by $\mathrm{n}: \hat{C} \rightarrow \bar{C}$ the normalization of the completion $\bar{C}$ of $C$ in $\overline{\text { Poly }_{3}} \simeq \mathbb{P}^{2}$. Pick any pair of positive integers $s=\left(s_{0}, s_{1}\right)$ and scale them such that 
Theorem 3.12 applies. This gives us a non-zero effective divisor $\mathrm{D}_{s}$ supported on $\hat{C} \backslash \mathrm{n}^{-1}(C)$. Replacing $s$ by a suitable multiple, we may suppose that it is very ample and pick a rational function $\phi$ on $\hat{C}$ whose divisor of poles and zeroes is greater or equal to $-\mathrm{D}_{s}$. Observe in particular that $\phi$ is a regular function on $\mathrm{n}^{-1}(C)$ that vanishes at finitely many points.

Consider the height $h_{s}$ induced by the semi-positive adelic metrics given by $g_{s, v}$, see Theorem 3.12. If $(c, a)$ is a point in $\mathrm{n}^{-1}(C)$ that is defined over a finite extension $\mathbb{K}$, denote by $\mathrm{O}(c, a)$ its orbit under the action of the absolute Galois group of $\mathbb{K}$, and by $\operatorname{deg}(c, a)$ the cardinality of this orbit. Fix a rational function $\phi$ as above that is not vanishing at $(c, a)$ (this exists since $-\mathrm{D}_{s}$ is very ample). Let $M_{\mathbb{K}}$ be the set of places of $\mathbb{K}$. By CL2, $\S 3.1 .3]$, since $\phi(c, a) \neq 0$ we have

$$
\begin{aligned}
h_{s}(c, a) & =\frac{1}{\operatorname{deg}(c, a)} \sum_{\mathrm{O}(c, a)} \sum_{v \in M_{\mathbb{K}}}-\log |\phi|_{s, v}\left(c^{\prime}, a^{\prime}\right) \\
& =\frac{1}{\operatorname{deg}(c, a)} \sum_{\mathrm{O}(c, a)} \sum_{v \in M_{\mathbb{K}}}\left(g_{s, v}-\log |\phi|_{v}\right)\left(c^{\prime}, a^{\prime}\right) \\
& =\frac{1}{\operatorname{deg}(c, a)} \sum_{\mathrm{O}(c, a)} \sum_{v \in M_{\mathbb{K}}} g_{s, v}\left(c^{\prime}, a^{\prime}\right) \geq 0
\end{aligned}
$$

where the last equality follows from the product formula.

We now estimate the total height of the curve $\hat{C}$ using [CL2, (1.2.6) \& (1.3.10)]. Choose any two meromorphic functions $\phi_{0}, \phi_{1}$ such that $\operatorname{div}\left(\phi_{0}\right)+\mathrm{D}_{s}$ and $\operatorname{div}\left(\phi_{1}\right)+\mathrm{D}_{s}$ are both effective with disjoint support included in $\mathrm{n}^{-1}(C)$. Let $\sigma_{0}$ and $\sigma_{1}$ be the associated sections of $\mathcal{O}_{\hat{C}}\left(\mathrm{D}_{s}\right)$. Let $\sum n_{i}\left[c_{i}, a_{i}\right]$ be the divisor of zeroes of $\sigma_{0}$, and $\sum n_{j}^{\prime}\left[c_{j}^{\prime}, a_{j}^{\prime}\right]$ be the divisor of zeroes of $\sigma_{1}$. Then

$$
\begin{aligned}
h_{s}(\hat{C}) & =\sum_{v \in M_{\mathbb{K}}}\left(\widehat{\operatorname{div}}\left(\sigma_{0}\right) \cdot \widehat{\operatorname{div}}\left(\sigma_{1}\right) \mid \hat{C}\right)_{v} \\
& =\sum_{i} n_{i} h_{s}\left(c_{i}, a_{i}\right)-\sum_{v \in M_{\mathbb{K}}} \int_{\hat{C}} \log \left|\sigma_{0}\right|_{s, v} \Delta g_{s, v} \\
& =\sum_{v \in M_{\mathbb{K}}} \int_{\hat{C}} g_{s, v} \Delta g_{s, v} \geq 0,
\end{aligned}
$$

where the third equality follows from Poincaré-Lelong formula and writing $\log \left|\sigma_{0}\right|_{s, v}=$ $\log |\phi|_{v}-g_{s, v}$ with $\phi \in \mathbb{K}(C)$ defining the section $\sigma_{0}$.

The formula for the height of a closed point implies that for all post-critically finite polynomials $P_{c_{n}, a_{n}}$ we have $h_{s}\left(c_{n}, a_{n}\right)=0$. Since PCF polynomials are Zariski dense in $C$, the essential minimum of $h_{s}$ is non-positive. By the arithmetic Hilbert-Samuel theorem

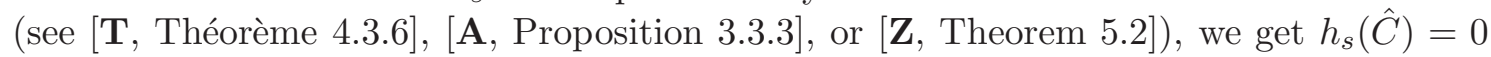
hence we may apply Thuillier-Yuan's theorem (see [T, $\mathbf{Y}]$ and [CL1, Théorème 4.2]). It follows that the sequence of probability measures $\mu_{n, v}$ that are equidistributed on $\mathrm{O}\left(c_{n}, a_{n}\right)$ in $\hat{C}^{v, \text { an }}$ converges to a probability measure $\mu_{\infty, v}$ that is proportional to $\Delta g_{s, v}$. We may thus write $\mu_{\infty, v}=w(s) \Delta g_{s, v}$ where $w(s) \in \mathbb{R}_{+}^{*}$ is equal to the inverse of the mass of $\Delta g_{s, v}$, i.e. to $\operatorname{deg}\left(\mathrm{D}_{s}\right)^{-1}$.

We now observe that $g_{s, v}$ is homogeneous in $s$ (i.e. $g_{\tau s, v}=\tau g_{s, v}$ for any $\tau \in \mathbb{R}_{+}^{*}$ ), and continuous with respect to this parameter. It follows that $w(s)$ is also continuous on $\left(\mathbb{R}_{+}^{*}\right)^{2}$, and $\mu_{\infty, v}=w(s) \Delta g_{s, v}$ for all $s \in\left(\mathbb{R}_{+}^{*}\right)^{2}$. 
From now on we fix an Archimedean place $v$. We shall treat the non-Archimedean case latter. We work in $\mathrm{n}^{-1}\left(C^{v \text {,an }}\right)$ which is the complement of finitely many points in the analytification of the smooth projective curve $\hat{C}^{v \text {,an }}$. To simplify notation we write $g_{0, v}, g_{1, v}$ instead of $g_{0, v} \circ \mathrm{n}, g_{1, v} \circ \mathrm{n}$. Recall that by [DF, Theorem 2.5] (see also [McM1, Theorem 2.2] or [De2, Theorem 1.1]) the equality $g_{0, v}=0$ on $\mathrm{n}^{-1}\left(C^{v, \text { an }}\right)$ implies $c_{0}$ to be persistently pre-periodic. Since we assumed that both $c_{0}$ and $c_{1}$ are not persistently pre-periodic, the functions $g_{0, v}$ and $g_{1, v}$ are not identically zero on $\mathrm{n}^{-1}\left(C^{v, \text { an }}\right)$.

Recall also that $g_{0, v}$ is harmonic where it is positive and that the support of $\Delta g_{0, v}$ is exactly the boundary of $\left\{g_{0, v}=0\right\}$ (see e.g. [DF, Proposition 6.7]). In particular $\Delta g_{0, v}$ is a non-zero positive measure, and its mass is finite by Proposition 3.6. Observe now that $g_{s, v} \rightarrow g_{0, v}$ uniformly on compact sets when $s$ tends to $(1,0)$, hence $\Delta g_{s, v} \rightarrow \Delta g_{0, v}$ and $\Delta g_{0, v}=t_{0} \mu_{\infty, v}$ for some positive $t_{0}$. In the same way, we get $\Delta g_{s, v} \rightarrow \Delta g_{1, v}$ as $s \rightarrow(0,1)$ which implies that the three positive measures $\mu_{\infty, v}, \Delta g_{0, v}$ and $\Delta g_{1, v}$ are proportional. We may thus find $s_{0}, s_{1}>0$ such that the function $H_{v}:=s_{0} g_{0, v}-s_{1} g_{1, v}$ is harmonic on $\mathrm{n}^{-1}\left(C^{v, \text { an }}\right)$.

Recall from [McM2 that the bifurcation locus of the family $P_{c, a}$ parameterized by $(c, a) \in \mathrm{n}^{-1}\left(C^{v \text {,an }}\right)$ is defined as the set where either $c_{0}$ or $c_{1}$ is unstable (or active in the terminology of $[\mathbf{D F}]$ ). It follows from $[\mathbf{D F}]$ that the bifurcation locus is equal to the union of the support of $\Delta g_{0, v}$ and $\Delta g_{1, v}$, hence to the support of $\mu_{\infty, v}$.

Suppose now that $H_{v}$ is not identically zero. Then this support is included in the locus $\left\{H_{v}=0\right\}$ which is real-analytic. This is impossible by McMullen's universality theorem, since the Hausdorff dimension of the bifurcation locus of any one-dimensional analytic family is equal to 2, see [McM3, Corollary 1.6].

We have proved that $s_{0} g_{0, v}=s_{1} g_{1, v}$ on $\mathrm{n}^{-1}\left(C^{v, \text { an }}\right)$ hence on $C^{v, \text { an }}$ for some positive real numbers $s_{0}, s_{1}>0$.

Since $g_{0, v}$ and $g_{1, v}$ are proportional, and $G_{v}=\max \left\{g_{0, v}, g_{1, v}\right\}$ is proper on $C^{v \text {,an }}$, it follows that $g_{0, v}$ is unbounded near any branch at infinity. By Proposition 3.6, $g_{0, v}$ admits an expansion of the form $g_{0, v}(t)=a(\mathfrak{c}) \log |t|^{-1}+O(1)$ with $a(\mathfrak{c}) \in \mathbb{Q}_{+}^{*}$ on the branch $\mathfrak{c}$ hence is locally superharmonic on that branch.

It follows that $\Delta g_{0, v}$ is a signed measure in $\hat{C}^{\text {an, } v}$ whose negative part is a divisor $\mathrm{D}_{0}$ with positive rational coefficients at any point of $\hat{C} \backslash \mathrm{n}^{-1}(C)$. The same being true for $\Delta g_{1, v}$, we conclude to the equality of divisors $s_{0} \mathrm{D}_{0}=s_{1} \mathrm{D}_{1}$. This implies that $s_{0} / s_{1}$ is rational, and we can assume $s_{0}$ and $s_{1}$ to be integers. This ends the proof of the first statement in the case the place is Archimedean.

Assume now that $v$ is non-Archimedean. One cannot copy the proof we gave in the Archimedean setting since we used the fact that $c_{0}$ is not persistently pre-periodic iff $\Delta g_{0, v}=0$, and McMullen's universality theorem, two facts that are valid only over $\mathbb{C}$.

Instead we apply Proposition 3.6. For each $s^{\prime}=\left(s_{0}^{\prime}, s_{1}^{\prime}\right)$ the function $g_{s^{\prime}, v}$ extends near any branch $\mathfrak{c}$ at infinity as an upper-semicontinuous function $\widehat{g_{s^{\prime}, v}}$ whose Laplacian puts some non-positive mass at $\mathfrak{c}$. When $s_{0}^{\prime}, s_{1}^{\prime} \neq 0$ then $g_{s^{\prime}, v}$ defines a positive continuous metric on $\mathcal{O}_{\hat{C}}\left(\mathrm{D}_{s}\right)$ hence $\Delta \widehat{g_{s^{\prime}, v}}\{\mathfrak{c}\}=-\operatorname{ord}_{\mathfrak{c}}\left(\mathrm{D}_{s}\right)<0$. This mass is in particular independent on the place. We get that

$$
-\Delta \widehat{g_{0, v}}\{\mathfrak{c}\} \geq \lim _{s \rightarrow(1,0)}-\Delta \widehat{g_{s, v}}\{\mathfrak{c}\}=\operatorname{ord}_{\mathfrak{c}}\left(\mathrm{D}_{0}\right)>0 .
$$

We infer that the mass of $\Delta g_{0, v}$ is equal to the degree of $\mathrm{D}_{0}$ hence is non-zero.

We may now argue as in the Archimedean case, and prove that $\Delta g_{0, v}$ and $\Delta g_{1, v}$ are proportional. The coefficient of proportionality is the only $t>0$ such that $\mathrm{D}_{0}=t \mathrm{D}_{1}$ hence 
$t=s_{0} / s_{1}$. Then $H_{v}:=s_{0} g_{0, v}-s_{1} g_{1, v}$ is harmonic on $C$ and bounded near any branch at infinity by Proposition 3.6, hence defines a harmonic function on the compact curve $\hat{C}^{\text {an }, v}$. It follows $H_{v}$ is a constant (in the non-Archimedean case by [ $\mathbf{T}$, Proposition 2.3.2]) which is necessarily zero since it is zero at all post-critically finite parameters.

We have completed the proof of Theorem 4.1 (1).

We mention here the following result that follows from the previous argument.

Corollary 4.2. - Let $C$ be an irreducible curve in Poly $_{3}$ defined over a number field $\mathbb{K}$ that contains infinitely many post-critically finite parameters and that neither $c_{0}$ nor $c_{1}$ is persistently pre-periodic. Pick an Archimedean place $v$.

Pick any sequence $X_{n} \subset C(\overline{\mathbb{K}})$ of Galois-invariant finite sets of postcritically finite parameters such that $X_{n} \neq X_{m}$ for $m \neq n$. Let $\mu_{n}$ be the measure equidistributed on $X_{n} \subset C^{v, \text { an }}$.

Then the sequence $\mu_{n}$ converges weakly to (a multiple of) $T_{\mathrm{bif}} \wedge[C]$ as $n \rightarrow \infty$.

Recall that $T_{\text {bif }}$ is defined as the $d d^{c}$ of the plurisubharmonic function $g_{0}+g_{1}$, and $[C]$ is the current of integration over the analytic curve $C^{v, \text { an }}$.

Proof. - Let $s_{0}, s_{1}>0$ be given by Theorem 4.1. As seen above, the sequence $\mu_{n}$ converges weakly towards $d d^{c} \max \left\{s_{0} \cdot g_{0}, s_{1} \cdot g_{1}\right\}=s_{0} \cdot d d^{c} g_{0}$ on $C^{v}$,an. It thus only remains to prove that $d d^{c}\left(\left.g_{0}\right|_{C^{v} \text {,an }}\right)=\kappa \cdot T_{\mathrm{bif}} \wedge[C]$ for some $\kappa>0$. Recall that $T_{\mathrm{bif}}=d d^{c}\left(g_{0}+g_{1}\right)$. By Theorem 4.1, on $C$,

$$
g_{0}+g_{1}=g_{0}+\frac{s_{1}}{s_{0}} \cdot g_{0}=\left(1+\frac{s_{0}}{s_{1}}\right) g_{0} .
$$

Let $\kappa:=1+\frac{s_{0}}{s_{1}}$. We thus have $d d^{c}\left(\left.g_{0}\right|_{C^{v} \text {,an }}\right)=\kappa^{-1} \cdot d d^{c}\left(\left.\left(g_{0}+g_{1}\right)\right|_{C^{v} \text {,an }}\right)$. Finally, since $g_{0}+g_{1}$ is continuous, we have $T_{\mathrm{bif}} \wedge[C]=d d^{c}\left(\left.\left(g_{0}+g_{1}\right)\right|_{C^{v} \text {,an }}\right)$, which ends the proof.

\subsection{Values of the Böttcher coordinates at critical points are proportional near infinity}

In this section, we prove Theorem 4.1 (2).

Let us fix a branch at infinity $\mathfrak{c}$ of an irreducible curve $C$ containing infinitely many PCF polynomials, and an isomorphism of complete local rings $\mathcal{O}_{\hat{C}, \mathfrak{c}} \simeq \mathbb{L}[[t]]$, such that $c(\mathrm{n}(t))=t^{-n}$, and $a(\mathrm{n}(t)) \in \mathcal{O}_{\mathbb{L}, S}((t))$ is an adelic series. Write $P_{t}=P_{c(\mathrm{n}(t)), a(\mathrm{n}(t))}$, and $\varphi_{t}=\varphi_{P_{t}}$.

By Lemma 3.8 there exists an integer $q \geq 1$ large enough such that $P_{t}^{q}\left(c_{0}\right)$ and $P_{t}^{q}\left(c_{1}\right)$ both lie in the domain of convergence of the Böttcher coordinate $\varphi_{t}$ for $t$ small enough, and (8) holds, i.e.

$$
\varphi_{t}\left(P_{t}^{q}\left(c_{\varepsilon}\right)\right)=\omega\left(P_{t}^{q}\left(c_{\varepsilon}\right)-\frac{c(\mathrm{n}(t))}{2}\right)+\Theta(t)
$$

where $\Theta$ is an adelic series vanishing at 0 .

We now fix a place $v$ and compute using Proposition 2.3 for $|t|_{v} \ll 1$. We get

$$
\begin{aligned}
\frac{\left|\varphi_{t}\left(P_{0}^{q}(t)\right)\right|_{v}^{s_{0}}}{\left|\varphi_{t}\left(P_{1}^{q}(t)\right)\right|_{v}^{s_{1}}} & =\frac{\exp \left(s_{0} \cdot g_{c(\mathrm{n}(t)), a(\mathrm{n}(t))}\left(P_{0}^{q}(t)\right)\right)}{\exp \left(s_{1} \cdot g_{c(\mathrm{n}(t)), a(\mathrm{n}(t))}\left(P_{1}^{q}(t)\right)\right)} \\
& =\frac{\exp \left(3^{q} s_{0} \cdot g_{0, v}(c(\mathrm{n}(t)), a(\mathrm{n}(t)))\right)}{\exp \left(3^{q} s_{1} \cdot g_{1, v}(c(\mathrm{n}(t)), a(\mathrm{n}(t)))\right.}=1,
\end{aligned}
$$

where the last equality follows from Theorem $4.1(1)$. 
Applying $(\star)$ in the case of an Archimedean place, we see that the complex analytic map

$$
t \longmapsto \frac{\left(\varphi_{t}\left(P_{0}^{q}(t)\right)\right)^{s_{0}}}{\left(\varphi_{t}\left(P_{1}^{q}(t)\right)\right)^{s_{1}}}
$$

has a modulus constant equal to 1 , hence is a constant, say $\zeta$. Since both power series $\varphi_{t}\left(P_{0}^{q}(t)\right)$ and $\varphi_{t}\left(P_{1}^{q}(t)\right)$ have their coefficients in $\mathcal{O}_{\mathbb{L}, S}$, we conclude that $\zeta \in \mathcal{O}_{\mathbb{L}, S}$. But $|\zeta|_{v}=1$ for all place $v$ over $\mathbb{L}$ by $(\star)$ hence it is a root of unity.

Note also that the equality $\varphi_{t}\left(P_{0}^{q}(t)\right)^{s_{0}}=\zeta \varphi_{t}\left(P_{1}^{q}(t)\right)^{s_{1}}$ holds as equality between adelic series, so that it is also true for analytic functions at any place.

To conclude the proof of Theorem 4.1. pick a place $v$ of $\mathbb{L}$ and consider the connected component $U$ of $\left\{g_{0, v}>\tau_{v} / s_{0}\right\}=\left\{g_{1, v}>\tau_{v} / s_{1}\right\}$ in $C^{v \text {,an }}$ whose closure in $\hat{C}$ contains $\mathfrak{c}$. We need to argue that $P_{c, a}^{q}\left(c_{0}\right)$ and $P_{c, a}^{q}\left(c_{1}\right)$ belongs to the domain of convergence of the Böttcher coordinate $\varphi_{v, c, a}$ for any $c, a \in U$.

Recall that $s_{0} g_{0, v}(c, a)=s_{1} g_{1, v}(c, a)$ for some positive integers $s_{0}, s_{1}$. It follows that

$$
\begin{aligned}
\min \left\{g_{c, a}\left(P_{c, a}^{q}\left(c_{0}\right)\right), g_{c, a}\left(P_{c, a}^{q}\left(c_{1}\right)\right)\right\} & =3^{q} \min \left\{g_{c, a}\left(c_{0}\right), g_{c, a}\left(c_{1}\right)\right\}> \\
3^{q} \min \left\{\frac{s_{0}}{s_{1}}, \frac{s_{1}}{s_{0}}\right\} \max \left\{g_{c, a}\left(c_{0}\right), g_{c, a}\left(c_{1}\right)\right\}> & \\
& G(c, a)+\max \left\{s_{0} g_{c, a}\left(c_{0}\right), s_{1} g_{c, a}\left(c_{1}\right)\right\}>G(c, a)+\tau_{v}
\end{aligned}
$$

for $q$ large enough and we conclude by Proposition 2.3.

The proof of Theorem 4.1 is now complete.

\section{Special curves having a periodic orbit with a constant multiplier}

In this section, we prove Theorem B.

Pick an integer $m \geq 1$, a complex number $\lambda \in \mathbb{C}$, and consider the set of polynomials $P_{c, a}$ that admits a periodic orbits of period $m$ and multiplier $\lambda$. It follows from Si1, p. 225] that this set is an algebraic curve in $\mathrm{Poly}_{3}$ (see also [Mi1, Appendix D], [BB2, Theorem 2.1] or [FG, $\S 6.2])$. Let us be more precise:

Theorem 5.1 (Silverman). - For any integer $m \geq 1$, there exists a polynomial $p_{m} \in$ $\mathbb{Q}[c, a, \lambda]$ with the following properties.

1. For any $\lambda \in \mathbb{C} \backslash\{1\}, p_{m}(c, a, \lambda)=0$ if and only if $P_{c, a}$ has a cycle of exact period $m$ and multiplier $\lambda$.

2. When $\lambda=1$, then $p_{m}(c, a, 1)=0$ if and only if there exists an integer $k$ dividing $m$ such that $P_{c, a}$ has a cycle of exact period $k$ whose multiplier is a primitive $m / k$-th root of unity.

We now come to the proof of Theorem B.

One implication is easy. For any integer $m \geq 1$, the curve $\operatorname{Per}_{m}(0)$ is contained in the union of the two curves $\left\{(c, a) \in \mathbb{C}^{2} ; P_{c, a}^{m}\left(c_{0}\right)=c_{0}\right\}$ and $\left\{(c, a) \in \mathbb{C}^{2} ; P_{c, a}^{m}\left(c_{1}\right)=c_{1}\right\}$. According to lemma 5.2 below, it contains infinitely many post-critically finite parameters.

Lemma 5.2. - Pick $n \geq 0, k>0$ and $i \in\{0,1\}$. Any irreducible component $C$ of the set $\left\{(c, a), P_{c, a}^{n+k}\left(c_{i}\right)=P_{c, a}^{n}\left(c_{i}\right)\right\}$ contains infinitely many post-critically finite parameters. 
Proof. - We argue over the complex numbers, and use the terminology and results from [DF. In particular, a critical point $c_{i}, i=0,1$ is said to be active at a parameter $(c, a)$ if the family of analytic functions $P_{c, a}^{n}\left(c_{i}\right)$ is normal in a neighborhood of $(c, a)$.

Suppose that $C$ is an irreducible component of the set

$$
\left\{(c, a), P_{c, a}^{n+k}\left(c_{i}\right)=P_{c, a}^{n}\left(c_{i}\right)\right\}
$$

where $n \geq 0, k>0$ and $i \in\{0,1\}$. To fix notation we suppose $i=0$. Observe that $g_{c, a}\left(c_{0}\right)=0$ on $C$, and since $G(c, a)=\max \left\{g_{c, a}\left(c_{0}\right), g_{c, a}\left(c_{1}\right)\right\}$ is a proper function on Poly 3 (see Proposition 2.1) it follows that $g_{c, a}\left(c_{1}\right)$ is also proper on $C$. In particular, $c_{1}$ has

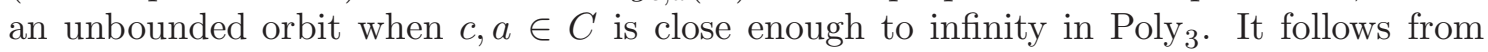
e.g. DF, Theorem 2.5] (which builds on McM1, Theorem 2.2]) that $c_{1}$ is active at at least one point $\left(c_{0}, a_{0}\right)$ on $C$. The arguments of [DF, Lemma 2.3] based on Montel's theorem show that $\left(c_{0}, a_{0}\right)$ is accumulated by parameters for which $c_{1}$ is pre-periodic to a repelling cycle, hence by post-critically finite polynomials. In particular, it contains infinitely many post-critically finite parameters.

For the converse implication, we proceed by contradiction and suppose that we can find a complex number $\lambda \neq 0$, an integer $m \geq 1$, and an irreducible component $C$ of $\operatorname{Per}_{m}(\lambda)$ containing infinitely many post-critically finite polynomials.

Observe that, whenever $0<|\lambda| \leq 1$, any parameter $(c, a) \in C \subset \operatorname{Per}_{m}(\lambda)$ has a nonrepelling cycle which is not super-attracting. In particular, at least one of its critical points has an infinite forward orbit (see e.g. [Mi2]). It follows that $\operatorname{Per}_{m}(\lambda)$ contains no post-critically finite parameter when $0<|\lambda| \leq 1$. This argument is however not sufficient to conclude in general. But we shall see that a combination of this argument applied at a place of residual characteristic 3 together with the study of the explosion of multipliers on a branch at infinity of $C$ gives a contradiction.

Proposition 5.3. - Suppose $C$ is an irreducible component of $\operatorname{Per}_{m}(\lambda)$ with $\lambda \in \mathbb{C}^{*}$ and $m \geq 1$ containing infinitely many post-critically finite polynomials. Then one of the two critical points is persistently periodic on $C$ and $\lambda$ is equal to the multiplier of a repelling periodic orbit of a post-critically finite quadratic polynomial.

We may thus assume that the curve $C$ is included in $\operatorname{Per}_{n}(0)$ for some integer $n \geq 1$ (possibly with $n \neq m$ ). Observe that the equation $P_{c, a}^{n}\left(c_{0}\right)=c_{0}\left(\operatorname{resp} . P_{c, a}^{n}\left(c_{1}\right)=c_{1}\right)$ is equivalent to the vanishing of a polynomial of the form $\sqrt{3}^{1-3^{n}} a^{3^{n}}+$ l.o.t (resp. $\sqrt{3}^{1-3^{n}}\left(a^{3}-\right.$ $\left.\frac{c^{3}}{6}\right)^{3^{n}}+$ l.o.t). It follows that the closure of $C$ in $\overline{\text { Poly }_{3}}$ intersects the line at infinity in a set included in $\{[1: 0: 0],[\zeta: 1: 0]\}$ with $\zeta^{3}=6$ (see also [BB2, Theorem 4.2]).

Consider the curve of unicritical polynomials $c_{0}=c_{1}$, which is defined by the equation $c=0$. It intersects the line at infinity at $[0: 1: 0]$, so that Bezout' theorem implies the existence of a parameter $(c, a) \in C$ which is unicritical.

We conjugate $P_{c, a}$ by a suitable affine map to a polynomial $Q(z)=z^{3}+t$. This unicritical polynomial has a periodic critical orbit. Proposition 5.4 below implies $|\lambda|_{v}<1$ at any place $v$ of residual characteristic 3 . By the previous proposition, $Q$ also has a periodic orbit whose multiplier is equal to the multiplier of a repelling orbit of a quadratic polynomial having a preperiodic critical point. Proposition 5.4 now gives $|\lambda|_{v}=1$ for this place, hence a contradiction.

The proof of Theorem $\mathrm{B}$ is complete. 
Proposition 5.4. - Suppose $Q(z)=z^{d}+t$ is a post-critically finite unicritical polynomial of degree $d \geq 2$, and let $\lambda \neq 0$ be the multiplier of some periodic orbit of $P$.

Then $\lambda$ belongs to some number field $\mathbb{K}$, and given any non-Archimedean place $v$ of $\mathbb{K}$ we have:

$-|\lambda|_{v}<1$ if the residual characteristic of $\mathbb{K}_{v}$ divides $d ;$

$-|\lambda|_{v}=1$ if the residual characteristic of $\mathbb{K}_{v}$ is prime to $d$.

Proof. - Since $Q$ is post-critically finite, $t$ satisfies a polynomial equation with integral coefficients hence belongs to a number field. Its periodic point are solutions of a polynomial of the form $Q^{n}(z)-z$ so that the periodic points of $Q$ and their multipliers also belong to a number field. We may thus fix a number field containing $t, \lambda$, and fix a place $v$ of $\mathbb{K}$ of residual characteristic $p \geq 2$.

Observe that the completion of the algebraic closure of the completion of $\mathbb{K}$ with respect to the norm induced by $v$ is a complete algebraically closed normed field isometric to the $p$-adic field $\mathbb{C}_{p}$. We consider the action of $Q$ on the Berkovich analytification of the affine plane over that field. To simplify notation we denote by $|\cdot|$ the norm on $\mathbb{C}_{p}$.

Suppose that $|t|>1$. Then we have $|Q(0)|=|t|>1$, and thus $\left|Q^{n}(0)\right|=\left|Q^{n-1}(0)\right|^{d}=$ $|t|^{d^{n}} \rightarrow \infty$ by an immediate induction. This would imply the critical point to have an infinite orbit contradicting our assumption that $Q$ is post-critically finite.

We thus have $|t| \leq 1$. This implies that any point having a bounded orbit lies in the closed unit ball $\{z,|z| \leq 1\}$. Indeed the same induction as before yields $|Q(z)|=|z|^{d}$ and $\left|Q^{n}(z)\right|=\left|Q^{n-1}(z)\right|^{d}=|z|^{d^{n}} \rightarrow \infty$ for any $|z|>1$.

Pick any periodic point $w$ of period $k$ with multiplier $\lambda=\left(Q^{k}\right)^{\prime}(w) \neq 0$. Observe that $Q^{\prime}(z)=d z^{d-1}$.

Suppose first that $p$ divides $d$ so that $|d|<1$. Since $\left|Q^{j}(w)\right| \leq 1$ for all $j \geq 0$ by what precedes, we have

$$
|\lambda|=\prod_{j=0}^{k-1}\left|Q^{\prime}\left(Q^{j}(w)\right)\right| \leq|d|^{k}<1 .
$$

Suppose now that $p$ is prime to $d$, hence $|d|=1$. Observe that one has $Q(B(z))=B(Q(z))$ for any $|z| \leq 1$ where $B(z)=\{w,|z-w|<1\}$ is the open ball of center $z$ and radius 1 . Since the critical point 0 has a finite orbit, two situations may arise.

Either $B(0)$ is strictly preperiodic, and thus cannot contain any periodic orbit. Or $B(0)$ is periodic, and is contained in the basin of attraction of some attracting periodic orbit. Since 0 has a finite orbit, it has to be periodic. In both cases this implies the orbit of $w$ to be included in the annulus $\{|z|=1\}$.

We thus have

$$
|\lambda|=\prod_{i=0}^{k-1}\left|Q^{\prime}\left(Q^{i}(w)\right)\right|=\prod_{i=0}^{k-1}\left|d\left(Q^{i}(w)\right)^{d-1}\right|=1,
$$

which concludes the proof.

Proof of Proposition 5.3, - Since $C$ contains infinitely many post-critically finite polynomials we may assume it is defined over a number field $\mathbb{K}$. Let $\hat{C}$ be the normalization of the completion of $C$ in $\overline{\mathrm{Poly}_{3}}$. Pick any branch $\mathfrak{c}$ of $C$ at infinity (i.e. a point in $\hat{C}$ which projects to the line at infinity in $\overline{\text { Poly }_{3}}$ ). By Proposition 3.5 we may choose an isomorphism of complete local rings $\widehat{\mathcal{O}_{\hat{C}, \mathfrak{c}}} \simeq \mathbb{L}[[t]]$ such that $c(\mathrm{n}(t)), a(\mathrm{n}(t))$ are adelic series, i.e. formal Laurent series with coefficients in $\mathcal{O}_{\mathbb{L}, S}((t))$ that are analytic at all places. 
In the remaining of the proof, we fix an Archimedean place, and embed $\mathbb{L}$ into the field of complex numbers (endowed with its standard norm). We may suppose $c(\mathrm{n}(t)), a(\mathrm{n}(t))$ are holomorphic in $0<|t|<\epsilon$ for some $\epsilon$, and meromorphic at 0 . We get a one-parameter family of cubic polynomials $P_{t}:=P_{c(\mathrm{n}(t)), a(\mathrm{n}(t))}$ parameterized by the punctured disk $\mathbb{D}_{\epsilon}^{*}=$ $\{0<|t|<\epsilon\}$.

Consider the subvariety $Z:=\left\{(z, t), P_{t}^{m}(z)=z\right\} \subset \mathbb{C} \times \mathbb{D}_{\epsilon}^{*}$. The projection map $Z \rightarrow \mathbb{D}_{\epsilon}^{*}$ is a finite cover which is unramified if $\epsilon$ is chosen small enough. By reducing $\epsilon$ if necessary, and replacing $t$ by $t^{N}$, we may thus assume that $Z \rightarrow \mathbb{D}_{\epsilon}^{*}$ is a trivial cover. In other words, there exists a meromorphic function $t \mapsto p(t)$ such that $P_{t}^{m}(p(t))=p(t)$ and $\left(P_{t}^{m}\right)^{\prime}(p(t))=\lambda$.

As in Section 3, we denote by $\mathrm{P}(z) \in \mathbb{C}((t))[z]$ the cubic polynomial induced by the family $P_{t}$. It induces a continuous map on the analytification $\mathbb{A}_{\mathbb{C}((t))}^{1, \text { an }}$, for which the point $\mathrm{p} \in \mathbb{A}^{1}(\mathbb{C}((t)))$ corresponding to $p(t)$ is periodic of period $m$ with multiplier $\left(\mathrm{P}^{m}\right)^{\prime}(\mathrm{p})=$ $\lambda$. Observe that $\mathrm{P}$ has two critical points $\mathrm{c}_{0}$ and $\mathrm{c}_{1}$ corresponding to the meromorphic functions 0 and $c(\mathrm{n}(t))$ respectively.

Lemma 5.5. - If $\mathrm{c}_{0}$ is not pre-periodic for $\mathrm{P}$, then $\left|\mathrm{P}^{q}\left(\mathrm{c}_{0}\right)\right|_{t}$ tends to infinity when $q \rightarrow$ $\infty$.

Proof. - Observe that our assumption is equivalent to the fact that $c_{0}$ is not persistently pre-periodic on $C$.

We claim that $g_{0}(t):=g_{P_{t}}\left(c_{0}\right)$ tends to infinity when $t \rightarrow 0$.

Suppose first that $c_{1}$ is persistently pre-periodic on $C$. Then the function $g_{1}$ is identically zero on $C$, so that $\left.G\right|_{C}=\left.\max \left\{g_{0}, g_{1}\right\}\right|_{C}=g_{0}$. Since $G$ is proper by Proposition 2.1, and $(c(\mathrm{n}(t)), a(\mathrm{n}(t)))$ tends to infinity in $\overline{\text { Poly }_{3}}$ when $t \rightarrow 0$, we conclude that $g_{0}(t) \rightarrow \infty$.

When $c_{1}$ is not persistently pre-periodic on $C$, the two functions $g_{0}(t)$ and $g_{1}(t):=$ $g_{P_{t}}\left(c_{1}\right)$ are proportional on $\mathfrak{c}$ by Theorem 4.1 (1). As before $\max \left\{g_{0}, g_{1}\right\} \rightarrow \infty$ as $t \rightarrow 0$ so that again $g_{0}(t) \rightarrow \infty$.

By Proposition [3.6, we can find $a>0$ such that $g_{0}\left(P_{t}\right)=a \log |t|^{-1}+O(1)$. And [DF, Lemma 6.4] implie:(3) the existence of a constant $C>0$ such that $g_{P_{t}}(z) \leq \log \max \{|z|,|c(\mathrm{n}(t))|,|a(\mathrm{n}(t))|\}+C$ for all $t$. Since $g_{P_{t}} \circ P_{t}=3 g_{P_{t}}$, we conclude that for all $q \geq 1$

$$
\log \max \left\{\left|P_{t}^{q}\left(c_{0}\right)\right|,|c(\mathrm{n}(t))|,|a(\mathrm{n}(t))|\right\} \geq 3^{q} g_{t}(0)-C=3^{q} a \log |t|^{-1}+O(1) .
$$

This implies $\left|P_{t}^{q}\left(c_{0}\right)\right|_{t} \geq 3^{q} a|t|_{t} \rightarrow \infty$ when $q \rightarrow \infty$ as required.

We continue the proof of Proposition [5.3. Suppose neither $c_{0}$ nor $c_{1}$ is persistently pre-periodic so that the previous lemma applies to both critical points. Translating its conclusion over the non-Archimedean field $\mathbb{C}((t))$, we get that $\mathrm{P}^{q}\left(\mathrm{c}_{0}\right)$ and $\mathrm{P}^{q}\left(\mathrm{c}_{1}\right)$ both tend to infinity when $q \rightarrow \infty$. We may thus apply [ $\mathbf{K}$, Theorem 1.1 (ii)], and $[\mathbf{K}$, Corollary 1.4] (which is directly inspired from a result of Bezivin). We conclude that all periodic cycles of $\mathrm{P}$ are repelling so that $\left|\left(\mathrm{P}^{m}\right)^{\prime}(\mathrm{p})\right|_{t}>1$. This contradicts $|\lambda|_{t}=1$.

Suppose next that $c_{0}$ is persistently pre-periodic (which implies $c_{1}$ not to be persistently pre-periodic). Then $c_{0}$ is pre-periodic whereas $c_{1}$ escapes to infinity by the previous lemma. Observe that if $c_{0}$ is eventually mapped to a point in the Julia set of $\mathrm{P}$, then $\mathbf{K}$, Theorem

\footnotetext{
$\overline{{ }^{(3)} \text { observe that }}$ the statement of the lemma is incorrectly stated in $[\mathbf{D F}$, and the constant $C$ is actually independent on $P$.
} 
1.1 (iii) (a)] combined with $[\mathbf{K}$, Corollary 1.4] implies that all cycles of $\mathrm{P}$ are repelling which gives a contradiction.

We can thus apply $\underline{\mathbf{K}}$, Theorem 1.1 (iii) (b)] to $\mathrm{P}$, and the preperiodic critical point $\mathrm{c}_{0}(=0)$ is contained in a closed ball $B=\left\{\mathbf{z} \in \mathbb{C}((t)),|\mathbf{z}|_{t} \leq r\right\}$ for some positive $r>0$ that is periodic of exact period $n$. Since $B$ is fixed by the polynomial $\mathrm{P}^{n}(z)=\sum_{j \geq 2} b_{j} z^{j}$ with coefficients $b_{j} \in \mathbb{C}((t))$, the radius $r$ satisfies an equation of the form $\left|b_{j}\right| r^{j}=r$ for some $j$ hence $r=|t|_{t}^{l}$ for some $l \in \mathbb{Q}$. To simplify the discussion to follow we do a suitable base change $t \rightarrow t^{N}$, and we conjugate $\mathrm{P}$ by the automorphism $\mathrm{z} \mapsto t^{-l} \mathrm{z}$ so that $B$ becomes the closed unit ball. Observe that 0 remains a critical point of $\mathrm{P}$ after this conjugacy.

Recall that the closed unit ball $B$ defines the Gauss point $x_{g} \in \mathbb{A}_{\mathbb{C}((t))}^{1, \text { an }}$ for which we have

$$
\left|\mathrm{Q}\left(\mathrm{x}_{g}\right)\right|:=\sup _{z \in B}|\mathrm{Q}(z)|_{t}=\max \left|q_{i}\right| \text { for all } \mathrm{Q}=\sum q_{i} z^{i} \in \mathbb{C}((t))[z] .
$$

Since $B$ is fixed by $\mathrm{P}^{n}$, it follows that $\mathrm{x}_{g}$ is also fixed by $\mathrm{P}^{n}$. This is equivalent to say that $\mathrm{P}^{n}$ can be written as $\mathrm{P}^{n}(z)=\sum_{i=1}^{3^{n}} a_{i} z^{i}$ where $\max \left|a_{i}\right|=1$.

For any $z \in \mathbb{C}((t))$ of norm 1 , denote by $\tilde{z}$ the unique complex number such that $|z-\tilde{z}|_{t}<1$.

Lemma 5.6. - We have $a_{1}=0,\left|a_{0}\right| \leq\left|a_{2}\right|=1$, and $\left|a_{i}\right|<1$ for all $i \geq 3$; and the complex quadratic polynomial $\tilde{P}(z):=\widetilde{a_{2}} z^{2}+\widetilde{a_{0}}$ has a preperiodic critical orbit.

Lemma 5.7. - The orbit of the periodic point $\mathrm{p}$ intersects the ball B.

Replacing $\mathrm{p}$ by its image by a suitable iterate of $\mathrm{P}$ we may suppose that it belongs to $B$, i.e. $|\mathrm{p}|_{t} \leq 1$. In fact we have $\left|\mathrm{P}^{i}(\mathrm{p})\right|_{t}=1$ for all $i \geq 0$.

Indeed if it were not the case, then the open unit ball would be periodic. Since it contains a critical point, it would be contained in the basin of attraction of an attracting periodic orbit which yields a contradiction.

Observe also that the period of $\mathrm{p}$ is necessarily a multiple of $n$, say $n k$ with $k \geq 1$.

To render the computation of the multiplier of $\mathrm{p}$ easier, we conjugate $\mathrm{P}^{n}$ by $z \mapsto a_{2} z$. Since $\left|a_{2}\right|=1$, we still have $|\mathbf{p}|_{t}=1$, and the equality $a_{2}=1$ is now satisfied.

By Lemma [5.6, we get $\sup _{B}|\mathrm{Q}|<1$ with $\mathrm{Q}:=\mathrm{P}^{n}-\widetilde{P}$, so that

$$
\left(\mathrm{P}^{n k}\right)^{\prime}(\mathrm{p})=\prod_{i=0}^{k-1}\left(\mathrm{P}^{n}\right)^{\prime}\left(\mathrm{P}^{n i}(\mathrm{p})\right)=\left(\widetilde{P}^{k}\right)^{\prime}(\widetilde{p}) .
$$

But the multiplier of $\mathrm{p}$ is equal to $\lambda \in \mathbb{C}$. Hence it is equal to the multiplier of a repelling periodic orbit of some quadratic polynomial (namely $\tilde{P}$ ) having a preperiodic critical orbit, as was to be shown.

Proof of Lemma 5.6. The point 0 is critical for P hence $a_{1}=0$.

Since the Gauss point is fixed by $\mathrm{P}^{n}$, we have $\max _{i \geq 2}\left|a_{i}\right|=1$. Let $d \geq 2$ be the maximum over all integers $i$ such that $\left|a_{i}\right|=1$. The number of critical points of $\mathrm{P}^{n}$ lying in the closed unit ball (counted with multiplicity) is precisely equal to $d-1$.

Since the exact period of $\mathrm{x}_{g}$ is $n$, and the other point escapes to infinity, the ball $B$ contains a unique critical point of $\mathrm{P}^{n}$ namely 0 . It follows that $d=2$, and $\left|a_{2}\right|=1>$ $\max _{i \geq 3}\left|a_{i}\right|$.

Finally 0 is preperiodic by $\mathrm{P}^{n}$, hence the complex quadratic polynomial $\widetilde{P^{n}}$ has a preperiodic critical orbit. 
Proof of Lemma 5.7 - Since the multiplier of $\mathrm{p}$ is $\lambda \in \mathbb{C}$, its $t$-adic norm is 1 , hence a small ball $U$ centered at $\mathrm{p}$ of positive radius is included in the filled-in Julia set of $\mathrm{P}$. By $[\mathbf{K}$, Corollary 4.8], $U$ is eventually mapped into $B$, hence the claim.

\section{A polynomial on a special curve admits a symmetry}

We fix $\mathbb{K}$ a number field, and $s_{0}, s_{1}$ two positive integers such that $s_{0}$ and $s_{1}$ are coprime. We shall say that a cubic polynomial $P:=P_{c, a}$ with $c, a$ in a finite extension $\mathbb{L}$ of $\mathbb{K}$ satisfies the condition $(\mathfrak{P})$ if the following holds:

$(\mathfrak{P} 1)$ For any place $v$ of $\mathbb{L}$, we have $s_{0} g_{P, v}\left(c_{0}\right)=s_{1} g_{P, v}\left(c_{1}\right)$.

$(\mathfrak{P} 2)$ Given any place $v$ of $\mathbb{L}$, if $\min \left\{g_{P, v}\left(P^{n}\left(c_{0}\right)\right), g_{P, v}\left(P^{n}\left(c_{1}\right)\right)\right\}>G_{v}(P)+\tau_{v}$ for some integer $n \geq 1$, then

$$
\frac{\varphi_{P, v}\left(P^{n}\left(c_{0}\right)\right)^{s_{0}}}{\varphi_{P, v}\left(P^{n}\left(c_{1}\right)\right)^{s_{1}}}
$$

is a root of unity lying in $\mathbb{K}$.

Recall the definition of the constant $\tau_{v}:=\tau\left(\mathbb{L}_{v}\right)$ from Proposition 2.3.

Observe that if the condition in $(\mathfrak{P} 2)$ never occurs, then the normalized heights by $P$ of both sequence of points $P^{n}\left(c_{0}\right)$ and $P^{n}\left(c_{1}\right)$ are bounded, hence $P$ is post-critically finite. We prove here the following

Theorem 6.1. - Suppose $P=P_{c, a}$ is a cubic polynomial defined over a number field $\mathbb{L}$ satisfying the assumptions $(\mathfrak{P})$ which is not post-critically finite and such that $\min \left\{g_{P, v}\left(P^{q}\left(c_{0}\right)\right), g_{P, v}\left(P^{q}\left(c_{1}\right)\right)\right\}>G_{v}(P)+\tau_{v}$ for some integer $q$ and some place $v$ of $\mathbb{L}$.

Then there exists a root of unity $\zeta \in \mathbb{K}$, an integer $q^{\prime} \leq C(\mathbb{K}, q)$, and an integer $m \geq 0$ such that the polynomial $Q(z):=\zeta P^{m}(z)+(1-\zeta) \frac{c}{2}$ commutes with all iterates $P^{k}$ such that $\zeta^{3^{k}}=\zeta$, and either $Q\left(P^{q^{\prime}}\left(c_{0}\right)\right)=P^{q^{\prime}}\left(c_{1}\right)$, or $Q\left(P^{q^{\prime}}\left(c_{1}\right)\right)=P^{q^{\prime}}\left(c_{0}\right)$.

Remark. - We shall prove along the way that there exists an integer $k \geq 1$ with $\zeta^{3^{k}}=\zeta$ so that the commutativity statement is non empty.

\subsection{Algebraization of adelic branches at infinity}

The material of this section is taken from $[\mathbf{X}]$. Let $\mathbb{K}$ be a number field. For any place $v$ on $\mathbb{K}$, denote by $\mathbb{K}_{v}$ the completion of $\mathbb{K}$ w.r.t. the $v$-adic norm.

We cover the line at infinity $H_{\infty}$ of the compactification of the affine space $\mathbb{A}_{\mathbb{K}}^{2}=$ Spec $\mathbb{K}[x, y]$ by $\mathbb{P}_{\mathbb{K}}^{2}$ by charts $U_{\alpha}=\operatorname{Spec} \mathbb{K}\left[x_{\alpha}, y_{\alpha}\right]$ centered at $\alpha \in H_{\infty}(\mathbb{K})$ such that $\alpha=\left\{\left(x_{\alpha}, y_{\alpha}\right)=(0,0)\right\}, H_{\infty} \cap U_{\alpha}=\left\{x_{\alpha}=0\right\}$, and $x_{\alpha}=1 / x, y_{\alpha}=y / x+c$ for some $c \in \mathbb{K}$ (or $x_{\alpha}=1 / y, y_{\alpha}=x / y$ ).

Fix $S$ a finite set of places of $\mathbb{K}$. By definition, an adelic branch $\mathfrak{s}$ at infinity defined over the ring $\mathcal{O}_{\mathbb{K}, S}$ is a formal branch based at a point $\alpha \in H_{\infty}(\mathbb{K})$ given in coordinates $x_{\alpha}, y_{\alpha}$ as above by a formal Puiseux series

$$
y_{\alpha}=\sum_{j \geq 1} a_{j} x_{\alpha}^{j / m} \in \mathcal{O}_{\mathbb{K}, S}\left[\left[x_{\alpha}^{1 / m}\right]\right]
$$

such that $\sum_{j \geq 1} a_{j} x^{j}$ is an adelic series.

Observe that for any place $v \notin S$, then the radius of convergence is a least 1 . In the sequel, we set $r_{\mathfrak{s}, \alpha, v}$ to be the minimum between 1 and the radius of convergence over $\mathbb{K}_{v}$ 
of this Puiseux series. Any adelic branch $\mathfrak{s}$ based at $\alpha$ at infinity thus defines an analytic curve in an (unbounded) open subset of $\mathbb{A}_{v}^{2, \text { an }}$ :

$$
Z^{v}(\mathfrak{s}):=\left\{\left(x_{\alpha}, y_{\alpha}\right) \in U_{\alpha}\left(\mathbb{K}_{v}\right) ; y_{\alpha}^{m}=\sum_{j \geq 1} a_{j} x_{\alpha}^{j}, 0<\left|x_{\alpha}\right|_{v}<r_{\mathfrak{s}, \alpha, v}\right\}
$$

Theorem 6.2 (Xie). - Suppose $\mathfrak{s}_{1}, \ldots, \mathfrak{s}_{l}$ are adelic branches at infinity, and let $\left\{B_{v}\right\}_{v \in M_{\mathbb{K}}}$ be a set of positive real numbers such that $B_{v}=1$ for all but finitely many places.

Assume that there exists a sequence of distinct points $p_{n}=\left(x_{n}, y_{n}\right) \in \mathbb{A}^{2}(\mathbb{K})$ such that for all $n$ and for each place $v \in M_{\mathbb{K}}$ then either we have $\max \left\{\left|x_{n}\right|_{v},\left|y_{n}\right|_{v}\right\} \leq B_{v}$ or $p_{n} \in \cup_{i=1}^{l} Z^{v}\left(\mathfrak{s}_{i}\right)$.

Then there exists an irreducible algebraic curve $Z$ defined over $\mathbb{K}$ such that any branch of $Z$ at infinity is contained in the set $\left\{\mathfrak{s}_{1}, \ldots, \mathfrak{s}_{l}\right\}$ and $p_{n}$ belongs to $Z(\mathbb{K})$ for all $n$ large enough.

\subsection{Construction of an invariant correspondence}

Our aim is to prove the following statement.

Theorem 6.3. - Suppose $P=P_{c, a}$ is a cubic polynomial satisfying the assumptions ( $\left.\mathfrak{P}\right)$. Then there exists a (possibly reducible) algebraic curve $Z_{P} \subset \mathbb{A}^{1} \times \mathbb{A}^{1}$ such that:

1. $\phi\left(Z_{P}\right)=Z_{P}$ with $\phi(x, y):=(P(x), P(y))$;

2. for all $n$ large enough, we have $\left(P^{n}\left(c_{0}\right), P^{n}\left(c_{1}\right)\right) \in Z_{P}$;

3. any branch at infinity of $Z_{P}$ is given by an equation $\varphi_{P}(x)^{s_{0}}=\zeta \cdot \varphi_{P}(y)^{s_{1}}$ for some root of unity $\zeta \in \mathbb{K}$.

Proof. - The proof is a direct application of Xie's theorem. Recall that the set $U_{\mathbb{K}}$ of roots of unity that is contained in the number field $\mathbb{K}$ is finite.

Recall that for each place $v$ over $\mathbb{L}$, we let $g_{P, v}:=\lim _{n} \frac{1}{3^{n}} \log ^{+}\left|P^{n}\right|_{v}$ be the Green function of $P$, and write $G_{v}(P)=\max \left\{g_{P, v}\left(c_{0}\right), g_{P, v}\left(c_{1}\right)\right\}$.

Lemma 6.4. - For any $\zeta \in U$, there exists an adelic branch $\mathfrak{c}_{\zeta}$ based at a point $q \in$ $H_{\infty}(\mathbb{L})$ such that for any place $v$ the analytic curve $Z^{v}\left(\mathfrak{c}_{\zeta}\right)$ is defined by the equation $\left\{\varphi_{P, v}(x)^{s_{0}}=\zeta \cdot \varphi_{P, v}(y)^{s_{1}}\right\}$ in the range $\min \left\{|x|_{v},|y|_{v}\right\}>\exp \left(G_{v}(P)+\tau_{v}\right)$.

Define $\left(x_{n}, y_{n}\right):=\left(P^{n}\left(c_{0}\right), P^{n}\left(c_{1}\right)\right) \in \mathbb{A}^{2}(\mathbb{L})$, and consider the family of all adelic curves $\mathfrak{c}_{\zeta}$ given by Lemma 6.4 for all $\zeta \in U_{\mathbb{K}}$. We shall first check that all hypothesis of Xie's theorem are satisfied.

To do so pick any integer $n$ and any place $v$ on $\mathbb{L}$. Suppose first that $g_{P, v}\left(c_{0}\right)=0$. Since $g_{P, v}\left(P^{n}\left(c_{0}\right)\right)=3^{n} g_{P, v}\left(c_{0}\right)=0$, we get $\left|x_{n}\right|_{v} \leq e^{C_{v}}=: B_{v}$ by Lemma 2.4. The same upper bound applies to $\left|y_{n}\right|_{v}$ since $g_{P, v}\left(c_{1}\right)=0$ by $(\mathfrak{P} 1)$ so that $\max \left\{\left|x_{n}\right|_{v},\left|y_{n}\right|_{v}\right\} \leq B_{v}$ in this case. Observe that $B_{v}=1$ for all but finitely many places $v$ of $\mathbb{L}$ by Lemma 2.4.

Suppose now that $g_{P, v}\left(c_{0}\right)>0$ so that $g_{P, v}\left(c_{1}\right)>0$ by $(\mathfrak{P} 1)$. Fix $N$ large enough such that $g_{P, v}\left(P^{N}\left(c_{0}\right)\right)>G_{v}(P)+\tau_{v}$ and $g_{P, v}\left(P^{N}\left(c_{1}\right)\right)>G_{v}(P)+\tau_{v}$. Then $P^{N}\left(c_{0}\right)$ and $P^{N}\left(c_{1}\right)$ lie in the domain of definition of the Böttcher coordinate by Proposition 2.3. Since

$$
g_{P, v}\left(P^{n}\left(c_{0}\right)\right)=3^{n-N} g_{P, v}\left(P^{N}\left(c_{0}\right)\right) \geq g_{P, v}\left(P^{N}\left(c_{0}\right)\right)>G_{v}(P),
$$


we may also evaluate $\varphi_{P}$ at $x_{n}$ for all $n \geq N$. The same holds for $y_{n}$ and we get that

$$
\frac{\varphi_{P}\left(x_{n}\right)^{s_{0}}}{\varphi_{P}\left(y_{n}\right)^{s_{1}}}
$$

is a root of unity $\zeta \in \mathbb{K}$ by $(\mathfrak{P} 2)$ hence $\left(x_{n}, y_{n}\right)$ belongs to $Z^{v}\left(\mathfrak{c}_{\zeta}\right)$ for all $n \geq N$.

Xie's theorem thus applies to the sequence $\left\{\left(x_{n}, y_{n}\right)\right\}_{n \geq N}$, and we get an irreducible curve $Z \subset \mathbb{A}^{1} \times \mathbb{A}^{1}$ that contains infinitely many points $\left(x_{n}, y_{n}\right)$ and such that each of its branch at infinity is equal to $\mathfrak{c}_{\zeta}$ for some $\zeta \in U_{\mathbb{K}}$.

Recall that $\phi(x, y)=(P(x), P(y))$, and pick any integer $n \geq 1$. Then $\phi^{n}(Z)$ is an irreducible curve defined over $\mathbb{L}$ whose branches at infinity are the images under $\phi^{n}$ of the branches at infinity of $Z$.

Fix $\zeta \in U_{\mathbb{K}}$ and pick $(x, y) \in Z^{v}\left(\mathfrak{c}_{\zeta}\right)$. Then $\left(x^{\prime}, y^{\prime}\right)=(P(x), P(y))$ satisfies

$$
\frac{\varphi_{P}\left(x^{\prime}\right)^{s_{0}}}{\varphi_{P}\left(y^{\prime}\right)^{s_{1}}}=\frac{\varphi_{P}(x)^{3 s_{0}}}{\varphi_{P}(y)^{3 s_{1}}}=\zeta^{3},
$$

hence $\phi\left(\mathfrak{c}_{\zeta}\right)=\mathfrak{c}_{\zeta^{3}}$. We conclude that any branch at infinity of $\phi^{n}(Z)$ is of the form $\mathfrak{c}_{\zeta}$ for some $\zeta \in U_{\mathbb{K}}$. Since two irreducible curves having a branch at infinity in common are equal, we see that $Z$ is pre-periodic for the morphism $\phi$ so that $\phi^{l+k}(Z)=\phi^{k}(Z)$ for some $l, k>0$. Setting $Z_{P}:=\cup_{j=k}^{l+k-1} \phi^{j}(Z)$, we obtain a (possibly reducible) curve defined over $\mathbb{L}$ such that $\phi\left(Z_{P}\right)=Z_{P}$ and $\left(x_{n}, y_{n}\right) \in Z_{P}$ for all $n \geq k$. This concludes the proof of the theorem.

Proof of Lemma 6.4 - Recall from Lemma 2.2 that

$$
\varphi_{P}(z)=\omega\left(z-\frac{c}{2}\right)+\sum_{k \geq 1} \frac{a_{k}}{z^{k}}
$$

is an adelic series at infinity in the sense of 3.1 , and therefore

$$
\varphi_{P}^{-1}(z)=\frac{1}{\omega} z+\frac{c}{2}+\sum_{k \geq 1} \frac{b_{k}}{z^{k}}
$$

too by Lemma 3.1. We may assume that $a_{k}, b_{k} \in \mathcal{O}_{\mathbb{K}, S}$. Recall from Proposition 2.3 that $\varphi_{P, v}$ induces an analytic isomorphism between $\left\{z, g_{P, v}(z)>G_{v}(P)+\tau_{v}\right\}$ and $\left\{z^{\prime},\left|z^{\prime}\right|_{v}>\right.$ $\left.\exp \left(G_{v}(P)+\tau_{v}\right)\right\}$. By Lemma 3.1 the formal map $\varphi_{P}^{-1}$ defines an adelic series at infinity in the terminology of 3.1 . For each place $v$, this series coincides with the inverse map of $\varphi_{P}$ on the complement of the closed disk of radius $\exp \left(G_{v}(P)+\tau_{v}\right)$ hence its domain of convergence is exactly $\left\{z^{\prime},\left|z^{\prime}\right|_{v}>\exp \left(G_{v}(P)+\tau_{v}\right)\right\}$.

It follows that

$$
Z_{v}:=\left\{(x, y), \varphi_{P}(x)^{s_{0}}=\zeta \varphi_{P}(y)^{s_{1}}\right\}
$$

defines an analytic curve in the domain $\min \left\{g_{P, v}(x), g_{P, v}(y)\right\}>G_{v}(P)+\tau_{v}$, whose image under the isomorphism $\left(x^{\prime}, y^{\prime}\right):=\left(\varphi_{P, v}(x), \varphi_{P, v}(y)\right)$ is given by

$$
Z_{v}^{\prime}:=\left\{\left(x^{\prime}, y^{\prime}\right),\left(x^{\prime}\right)^{s_{0}}=\zeta\left(y^{\prime}\right)^{s_{1}}\right\}
$$

where $\min \left\{\left|x^{\prime}\right|_{v},\left|y^{\prime}\right|_{v}\right\}>\exp \left(G_{v}(P)+\tau_{v}\right)$.

Pick any $\xi \in \overline{\mathbb{Q}}$ such that $\xi^{s_{1}} \zeta=1$. Let $\mathfrak{c}_{\zeta}$ be the adelic branch at infinity defined by the formal Laurent series $\left(\varphi_{P}^{-1}\left(t^{-s_{1}}\right), \varphi_{P}^{-1}\left(\xi t^{-s_{0}}\right)\right)$. For any place $v$, the analytic curve $Z^{v}\left(\mathfrak{c}_{\zeta}\right)$ is included in $Z_{v}$. Since $s_{0}$ and $s_{1}$ are coprime, for any pair $\left(x^{\prime}, y^{\prime}\right)$ with $\left(x^{\prime}\right)^{s_{0}}=\zeta\left(y^{\prime}\right)^{s_{1}}$ and $\min \left\{\left|x^{\prime}\right|_{v},\left|y^{\prime}\right|_{v}\right\}>\exp \left(G_{v}(P)+\tau_{v}\right)$, there exists $0<|t|_{v}<\exp \left(-\frac{G_{v}(P)+\tau_{v}}{\min \left\{s_{0}, s_{1}\right\}}\right)$ such that $x^{\prime}=t^{-s_{1}}$ and $y^{\prime}=\xi t^{-s_{0}}$. 
This proves that $Z^{v}\left(\mathfrak{c}_{\zeta}\right)=Z_{v}$ for all place as required.

\subsection{Invariant correspondences are graphs}

Let $Z_{0}, \ldots, Z_{p-1}$ be the irreducible components of $Z_{P}$ such that $\phi\left(Z_{i}\right)=Z_{i+1}$ (the index computed modulo $p$ ). Since we assumed $P$ not to be post-critically finite, it is non-special in the sense of $[\mathbf{P}$. We may thus apply Theorem 4.9 of op. cit. (or [MS , Theorem 6.24]) to the component $Z_{0}$ of $Z_{P}$ that is $\phi^{p}$-invariant. It implies that after exchanging $x$ and $y$ if necessary, $Z_{0}$ is the graph of a polynomial map, i.e. $Z_{0}=\{(Q(t), t)\}$ for some $Q \in \mathbb{L}[t]$ such that $Q \circ P^{p}=P^{p} \circ Q$. Observe that by [J] the two polynomials $P$ and $Q$ share a common iterate when $\operatorname{deg}(Q) \geq 2$ since we assumed $P$ not to be post-critically finite.

We now work at an Archimedean place. Recall that the branch at infinity of $Z_{0}$ is of the form $\varphi_{P}(x)^{s_{0}}=\zeta \varphi_{P}(y)^{s_{1}}$ for some $\zeta \in U_{\mathbb{K}}$. Since $s_{0}$ and $s_{1}$ are coprime, it follows that $s_{0}=1$ and $s_{1}=\operatorname{deg}(Q)$, and therefore $s_{1}$ is a power of 3 , say $s_{1}=3^{m}$. We get

$$
\varphi_{P}(Q(t))=\zeta \varphi_{P}(t)^{3^{m}}=\zeta \varphi_{P}\left(P^{m}(t)\right) .
$$

for all $t$ of large enough norm. By Lemma 2.2, we get that $\varphi_{P}(t)=\omega\left(t-\frac{c}{2}\right)+o(1)$ so that

$$
\omega\left(Q(t)-\frac{c}{2}\right)=\omega \zeta\left(P^{m}(t)-\frac{c}{2}\right)+o(1)
$$

which implies $Q(t):=\zeta P^{m}(t)+(1-\zeta) \frac{c}{2}$ since a polynomial which tends to 0 at infinity is identically zero.

At this point, recall our assumption that $\min \left\{g_{P, v}\left(P^{q}\left(c_{0}\right)\right), g_{P, v}\left(P^{q}\left(c_{1}\right)\right)\right\}>G_{v}(P)+\tau_{v}$ for some integer $q$ and some place $v$ of $\mathbb{L}$. Then by $(\mathfrak{P} 2) \varphi_{P}\left(P^{q}\left(c_{0}\right)\right)^{s_{0}}=\xi \varphi_{P}\left(P^{q}\left(c_{1}\right)\right)^{s_{1}}$ for some root of unity $\xi \in \mathbb{K}$ which implies $\varphi_{P}\left(P^{q+n}\left(c_{0}\right)\right)=\xi^{3^{n}} \varphi_{P}\left(P^{q+n}\left(c_{1}\right)\right)^{3^{m}}$. Since for some $n$ large enough the point $\left(P^{q+n}\left(c_{0}\right), P^{q+n}\left(c_{1}\right)\right)$ belongs to $Z_{0}$, we get $\xi^{3^{n}}=\zeta$. Now observe that the least integer $n$ such that $\xi^{3^{n}}=\zeta$ is less that the cardinality of $U_{\mathbb{K}}$. We get the existence of $q^{\prime} \leq C(\mathbb{K}, q)$ such that $\varphi_{P}\left(P^{q^{\prime}}\left(c_{0}\right)\right)=\zeta \varphi_{P}\left(P^{q^{\prime}}\left(c_{1}\right)\right)^{3^{m}}$. Since $\varphi_{P}$ is injective, the equation (13) shows that $P^{q^{\prime}}\left(c_{0}\right)=Q\left(P^{q^{\prime}}\left(c_{1}\right)\right)$.

Observe that $\zeta^{3^{p}}=\zeta$. Indeed, since $Z_{0}$ is $\phi^{p}$-invariant and since $\phi\left(\mathfrak{c}_{\zeta}\right)=\mathfrak{c}_{\zeta^{3}}$, we get $\mathfrak{c}_{\zeta^{3}}=\mathfrak{c}_{\zeta}$, hence $\zeta^{3^{p}}=\zeta$.

We now pick any integer $k \geq 1$ such that $\zeta^{3^{k}}=\zeta$. Then for all $t$ large enough, we have

$$
\varphi_{P}\left(Q \circ P^{k}(t)\right)=\zeta \varphi_{P}\left(P^{k}(t)\right)^{3^{m}}=\zeta \varphi_{P}^{3^{m+k}}(t)
$$

whereas

$$
\varphi_{P}\left(P^{k} \circ Q(t)\right)=\varphi_{P}(Q(t))^{3^{k}}=\zeta^{3^{k}} \varphi_{P}^{3^{k+m}}(t) .
$$

Since $\varphi_{P}$ is injective on a neighborhood of $\infty$, and since $\zeta^{3^{k}}=\zeta$ by assumption, we conclude that $Q \circ P^{k}=P^{k} \circ Q$.

This concludes the proof of Theorem 6.3.

\section{Classification of special curves}

In this section, we prove Theorems $\mathrm{C}$ and $\mathrm{A}$.

Before starting the proofs, let us introduce some notation. Pick $q, m \geq 0$ and $\zeta$ a root of unity. We let $Z(q, m, \zeta)$ be the algebraic set of those $(c, a) \in \mathbb{A}^{2}$ such that the polynomial 
$Q_{c, a}:=\zeta P_{c, a}^{m}+(1-\zeta) \frac{c}{2}$ commutes with all iterates $P_{c, a}^{k}$ of $P_{c, a}$ such that $\zeta^{3^{k}}=\zeta$, and either $Q_{c, a}\left(P_{c, a}^{q}\left(c_{0}\right)\right)=P_{c, a}^{q}\left(c_{1}\right)$, or $Q_{c, a}\left(P_{c, a}^{q}\left(c_{1}\right)\right)=P_{c, a}^{q}\left(c_{0}\right)$.

Observe that, when $m \geq 1, Q$ has degree $3^{m}$ and when $(c, a)$ belongs to a fixed normed field $K$ then the Green function $g_{Q}:=\lim _{n} \frac{1}{3^{n m}} \log ^{+}\left|Q^{n}\right|$ is equal to $g_{c, a}$. Indeed since $Q$ and $P^{k}$ commute they have the same filled-in Julia set, $\mathcal{K}_{Q}$ coincides with the filled-in Julia set $\mathcal{K}_{P}$ of $P$. And $g_{P}$ (resp. $g_{Q}$ ) is the unique continuous sub-harmonic function $g$ on $\mathbb{A}_{K}^{1, \text { an }}$ that is zero on $\mathcal{K}_{P}$, harmonic outside, with a logarithmic growth at infinity $g_{P}(z)=\log |z|+O(1)\left(\right.$ resp. $\left.g_{Q}(z)=\log |z|+O(1)\right)$. As $\mathcal{K}_{P}=\mathcal{K}_{Q}$, this gives $g_{c, a}=g_{Q}$.

\subsection{Proof of Theorem C}

The implication $(1) \Rightarrow(3)$ is exactly point 1 . of Theorem 4.1 .

The implication $(3) \Rightarrow(4)$ follows from Corollary 4.2 when $s_{0}$ and $s_{1}$ are both nonzero. Indeed, by assumption the critical point $c_{0}$ is pre-periodic for $P_{c, a}$ if and only if $c_{1}$ is. Moreover we observe that $c_{0}$ is active at at least one parameter of $C$, since the function $G$ is proper, hence that the set for which it is pre-periodic is infinite, by e.g. DF, Lemma $2.3]$.

When $s_{1}=0$, then $g_{0, v} \equiv 0$ on $C$ at all places. By [DF, Theorem 2.5] there exist $n>0$ and $k \geq 0$ such that $C$ is an irreducible component of $\left\{(c, a) \in \mathbb{A}^{2} ; P_{c, a}^{n+k}\left(c_{0}\right)=P_{c, a}^{k}\left(c_{0}\right)\right\}$. By Theorem 3.12 (applied to arbitrary weights) the family of functions $\left\{g_{1, v}\right\}_{v \in M_{\mathbb{K}}}$ induces a semi-positive adelic metric on some ample line bundle on the normalization of the completion of $C$ so that Thuillier-Yuan's theorem applies. This gives (4) by observing that $g_{0}+g_{1}=g_{1}$. The case $s_{0}=0$ is treated similarly.

The implication $(4) \Rightarrow(1)$ is obvious.

To prove $(2) \Rightarrow(1)$, we observe that if $c_{0}$ is not persistently pre-periodic on $C$ then it is active at at least one parameter by [DF, Theorem 2.5] and that the set of parameters for which it is pre-periodic is infinite by e.g. [DF, Lemma 2.3].

We now prove $(3) \Rightarrow(2)$. We suppose $c_{0}$ is not persistently pre-periodic on $C$. Pick some parameter $(c, a) \in C$ and suppose $c_{0}$ is pre-periodic. We need to show that $P_{c, a}$ is post-critically finite.

Since $c_{0}$ is not persistently pre-periodic on $C$ we have $s_{1} \neq 0$ (again by $\mathbf{D F}$, Theorem 2.5] applied at any Archimedean place). In the case $s_{0}=0$ then $c_{1}$ is persistently preperiodic and $P_{c, a}$ is clearly post-critically finite. We may thus assume that $s_{0}$ and $s_{1}$ are both non-zero and the functions $g_{0, v}, g_{1, v}$ vanish on the same set in $C^{v \text {,an }}$ for any place $v$ of $\mathbb{K}$. Observe that $c_{0}$ being pre-periodic implies $(c, a)$ to be defined over a number field. It follows that for all the Galois-conjugates $\left(c^{\prime}, a^{\prime}\right)$ of $(c, a)$ (over the defining field $\mathbb{K}$ of the curve $C$ ) we have $G_{v}\left(c^{\prime}, a^{\prime}\right):=\max \left\{g_{0, v}\left(c^{\prime}, a^{\prime}\right), g_{1, v}\left(c^{\prime}, a^{\prime}\right)\right\}=0$. It follows from [I] or [FG, Theorem 3.2] that $P_{c, a}$ is post-critically finite.

Let us now prove $(3) \Rightarrow(5)$. We suppose $C$ is special. If either $c_{0}$ or $c_{1}$ is persistently pre-periodic in $C$, the assertion (5) holds true with $\zeta=1$ and $i=j$ by [DF, Theorem 2.5].

Assume from now on that we are not in this case. Replacing $\mathbb{K}$ by a finite extension we may assume that all roots of unity $\zeta$ appearing in Theorem 6.1 2. belong to $\mathbb{K}$, since there are at most the number of branches at infinity of $C$ of such roots of unity.

Let $\mathcal{B}$ be the set of all $(c, a) \in C(\mathbb{L})$ where $\mathbb{L}$ is a finite extension of $\mathbb{K}$ such that $P_{c, a}$ is not post-critically finite. Given a place $v$ of $\mathbb{K}$ we also define the subset $\mathcal{B}_{v}$ of $\mathcal{B}$ of parameters $c, a$ such that $g_{0, v}(c, a)>0$. This set is infinite since post-critically finite polynomials form a bounded set in $C^{v \text {,an }}$. 
Lemma 7.1. - For any $(c, a) \in \mathcal{B}$, the polynomial $P_{c, a}$ satisfies $(\mathfrak{P} 1)$ and $(\mathfrak{P} 2)$.

Pick $q$ large enough such that $3^{q}>\max \left\{s_{0} / s_{1}, s_{1} / s_{0}\right\}$, and fix a place $v$ of residual characteristic $\geq 5$. Now choose any $(c, a) \in \mathcal{B}_{v}$. Then $g_{1, v}(c, a)$ is also positive and $\min \left\{g_{c, a, v}\left(P^{q}\left(c_{0}\right)\right), g_{c, a, v}\left(P^{q}\left(c_{1}\right)\right)\right\}>G_{v}(c, a)$ so that Theorem 6.1 applies by the previous lemma. We get a positive integer $q^{\prime}$ (bounded from above by a constant $C$ depending only on $\mathbb{K}$ and $q)$, a root of unity $\zeta \in \mathbb{K}$ and an integer $m \geq 0$ such that $(c, a) \in Z\left(q^{\prime}, m, \zeta\right)$.

Since $g_{Q}=g_{P}$, and $Q\left(P^{q^{\prime}}\left(c_{0}\right)\right)=P^{q^{\prime}}\left(c_{1}\right)$ we have

$$
3^{m} g_{P, v}\left(P^{q^{\prime}}\left(c_{0}\right)\right)=g_{P, v}\left(Q\left(P^{q^{\prime}}\left(c_{0}\right)\right)\right)=g_{P, v}\left(P^{q^{\prime}}\left(c_{1}\right)\right)
$$

so that $3^{m}=\frac{s_{0}}{s_{1}}$. We conclude that the algebraic set consisting of the union of the curves $Z\left(q^{\prime}, m, \zeta\right)$ with $3^{m}=\frac{s_{0}}{s_{1}}, q^{\prime} \leq C$ and $\zeta$ ranging over all roots of unity lying in $\mathbb{K}$ contains $\mathcal{B}_{v}$.

It follows that $C$ is an irreducible component of one of these curves.

To end the proof of the theorem, we are left with proving $(5) \Rightarrow(3)$. Suppose that $C$ is an irreducible component of $Z(m, q, \zeta)$ for some $m \geq 0$ and $q \geq 0$ and some root of unity $\zeta$. Observe that $Z(q, m, \zeta)$ hence $C$ are defined over a number field say $\mathbb{K}$.

When $m \geq 1$, then for all place $v$ of that number field we have $g_{Q_{c, a}, v}=g_{P_{c, a}, v}$ for all $(c, a) \in C(\mathbb{L})$ for some finite extension $\mathbb{L}$ of $\mathbb{K}$. In particular $Q_{c, a}\left(P_{c, a}^{q}\left(c_{i}\right)\right)=P_{c, a}^{q}\left(c_{j}\right)$ implies $3^{m} g_{i, v}(c, a)=g_{j, v}(c, a)$ which proves (3) (with $s_{0}=0$ or $s_{1}=0$ if $i=j$ ). When $m=0$ and $\zeta \neq 1$ and $C \neq\{c=0\}$, then $Q_{c, a}\left(c_{0}\right)=(1-\zeta) c / 2 \neq 0$ hence $i \neq j$. It follows that $g_{P_{c, a}, v} \circ Q_{c, a}=g_{P_{c, a}, v}$ hence $g_{0, v}=g_{1, v}$. When $C=\{c=0\}$, then $c_{0}=c_{1}$ so that again $g_{0, v}=g_{1, v}$. Finally when $m=0$ and $\zeta=1$, then $i \neq j$ and $P_{c, a}^{q}\left(c_{0}\right)=P_{c, a}^{q}\left(c_{1}\right)$ hence $g_{0, v}=g_{1, v}$ at all places.

Proof of Lemma 7.1. - Pick $(c, a) \in \mathcal{B}$. By the point 1. of Theorem 4.1, $P_{c, a}$ satisfies $(\mathfrak{P} 1)$. To check $(\mathfrak{P} 2)$, we need to introduce a few sets. Fix any place $v$ of $\mathbb{K}$, and for any integer $n \geq 0$, define the open subset of $C^{v \text {,an }}$

$$
\Omega_{n, v}:=\left\{\left(c^{\prime}, a^{\prime}\right), \min \left\{g_{c^{\prime}, a^{\prime}, v}\left(P_{c^{\prime}, a^{\prime}}^{n}\left(c_{0}\right)\right), g_{c^{\prime}, a^{\prime}, v}\left(P_{c^{\prime}, a^{\prime}}^{n}\left(c_{1}\right)\right)\right\}>G_{v}\left(c^{\prime}, a^{\prime}\right)+\tau_{v}\right\} .
$$

On $\Omega_{n, v}$ one can define the analytic map

$$
M_{n}\left(c^{\prime}, a^{\prime}\right):=\frac{\varphi_{c^{\prime}, a^{\prime}}\left(P_{c^{\prime}, a^{\prime}}^{n}\left(c_{0}\right)\right)^{s_{0}}}{\varphi_{c^{\prime}, a^{\prime}}\left(P_{c^{\prime}, a^{\prime}}^{n}\left(c_{1}\right)\right)^{s_{1}}} .
$$

Observe that $\Omega_{n+1, v} \subset \Omega_{n, v}$, and $M_{k+l}\left(c^{\prime}, a^{\prime}\right)=M_{k}\left(c^{\prime}, a^{\prime}\right)^{3^{l}}$ on $\Omega_{k, v}$ for all integers $k, l \geq 0$. We also define the increasing sequence of open sets

$$
U_{n, v}:=\left\{\left(c^{\prime}, a^{\prime}\right), G_{v}\left(c^{\prime}, a^{\prime}\right)>\frac{\tau_{v}}{3^{n}-1}\right\} \subset C^{v, \text { an }} .
$$

Since $G_{v}$ is subharmonic and proper on $C^{v \text {,an }}$, the set $U_{n, v}$ contains no bounded component by the maximum principle.

Lemma 7.2. - Suppose $3^{r} \geq \max \left\{s_{0} / s_{1}, s_{1} / s_{0}\right\}$. Then we have $\Omega_{n, v} \subset U_{n, v}$ and $U_{n, v} \subset$ $\Omega_{n+r, v}$.

By the point 2. of Theorem 4.1, one can find two integers $q \geq 1$ and $N \geq 1$ such that $M_{q}$ is well-defined and constant equal to a root of unity lying in $\mathbb{K}$ in each component of $U_{N, v}$.

Let $V$ be the connected component of $\Omega_{n, v}$ containing $(c, a)$. This open set might or might not be bounded. By the previous lemma, if $n \geq \max \{r+N, q\}$, then $U_{n-r, v} \subset \Omega_{n, v}$ 
so that $M_{n}$ is well-defined on $U_{n-r, v}$. Since all components of $U_{n-r, v}$ are unbounded, and $M_{n}=M_{q}^{3^{n-q}}$ in $U_{N, r}$, we conclude that $M_{n}$ is locally constant in $U_{n-r, v}$ (hence on $V$ ) equal to a root of unity lying in $\mathbb{K}$.

When $n \leq n_{0}=\max \{r+N, q\}$, then $\left(M_{n}\right)^{3^{n_{0}-n}}=M_{n_{0}}$ which we know is constant in $V$ equal to a root of unity lying in $\mathbb{K}$. We conclude that $M_{n}$ is constant on $V$ equal to a root of unity lying in a fixed extension $\mathbb{K}^{\prime}$ of $\mathbb{K}$ that only depends on the constants $r, N$ and $q$. Since these constants are in turn independent of the place $v$, we conclude the proof of the lemma replacing $\mathbb{K}$ by $\mathbb{K}^{\prime}$.

Proof of Lemma 7.2 - Pick $\left(c^{\prime}, a^{\prime}\right) \in \Omega_{n, v}$. We may suppose that $G_{v}\left(c^{\prime}, a^{\prime}\right)=g_{c^{\prime}, a^{\prime}, v}\left(c_{0}\right)$ so that $G_{v}\left(c^{\prime}, a^{\prime}\right)=g_{c^{\prime}, a^{\prime}, v}\left(c_{0}\right)=\frac{1}{3^{n}} g_{c^{\prime}, a^{\prime}, v}\left(P_{c^{\prime}, a^{\prime}}^{n}\left(c_{0}\right)\right)>\frac{1}{3^{n}}\left(G_{v}\left(c^{\prime}, a^{\prime}\right)+\tau_{v}\right)$ which implies $\left(c^{\prime}, a^{\prime}\right) \in U_{n, v}$.

Conversely suppose $\left(c^{\prime}, a^{\prime}\right) \in U_{n, v}$. As before we may suppose that $G_{v}\left(c^{\prime}, a^{\prime}\right)=$ $g_{c^{\prime}, a^{\prime}, v}\left(c_{0}\right)$ so that

$$
\begin{aligned}
g_{c^{\prime}, a^{\prime}, v}\left(P^{n+r}\left(c_{0}\right)\right) \geq g_{c^{\prime}, a^{\prime}, v}\left(P^{n}\left(c_{0}\right)\right) & =3^{n} g_{c^{\prime}, a^{\prime}, v}\left(c_{0}\right)= \\
3^{n} G_{v}\left(c^{\prime}, a^{\prime}\right) & =G_{v}\left(c^{\prime}, a^{\prime}\right)+\left(3^{n}-1\right) G_{v}\left(c^{\prime}, a^{\prime}\right)>G_{v}\left(c^{\prime}, a^{\prime}\right)+\tau_{v} .
\end{aligned}
$$

Similarly we have

$$
g_{c^{\prime}, a^{\prime}, v}\left(P^{n+r}\left(c_{1}\right)\right)=3^{n+r} \frac{s_{0}}{s_{1}} g_{c^{\prime}, a^{\prime}, v}\left(c_{1}\right) \geq 3^{n} g_{c^{\prime}, a^{\prime}, v}\left(c_{0}\right)>G_{v}\left(c^{\prime}, a^{\prime}\right)+\tau_{v}
$$

hence $\left(c^{\prime}, a^{\prime}\right) \in \Omega_{n+r, v}$.

\subsection{Proof of Theorem $\mathbf{A}$}

According to the implication $(1) \Rightarrow(5)$ of Theorem $[$, any irreducible algebraic curve $C$ of Poly $_{3}$ containing infinitely many post-critically finite polynomials is a component of some $Z(q, m, \zeta)$ so that Theorem $\mathrm{A}$ reduces to the following.

\section{Proposition 7.3.}

1. The set $Z(q, m, 1)$ is equal to the union $\left\{P_{c, a}^{m+q}\left(c_{1}\right)=P_{c, a}^{q}\left(c_{0}\right)\right\} \cup\left\{P_{c, a}^{m+q}\left(c_{0}\right)=\right.$ $\left.P_{c, a}^{q}\left(c_{1}\right)\right\}$, hence contains an algebraic curve. Moreover, one has $Z(1,0,1)=$ $Z(0,0,1)$.

2. The set $Z(q, m,-1)$ is infinite if and only if $m=0$, and we have $Z(q, 0,-1)=$ $\left\{12 a^{3}-c^{3}-6 c=0\right\}$ for any $q \geq 0$.

3. if $\zeta^{2} \neq 1$, the set $Z(q, m, \zeta)$ is finite.

We shall rely on the following observation. Denote by $\operatorname{Crit}(P)$ the set of critical points of the polynomial $P$.

Lemma 7.4. - Pick any $(c, a) \in Z(q, m, \zeta)$, and suppose that

$$
Q_{c, a}=\zeta P_{c, a}^{m}+(1-\zeta) \frac{c}{2}
$$

is a polynomial that commutes with $P_{c, a}^{k}$ and $\zeta$ is a $\left(3^{k}-1\right)$-root of unity. Then we have

$$
Q_{c, a}\left(\operatorname{Crit}\left(P_{c, a}^{k+m}\right)\right)=Q_{c, a}\left(\operatorname{Crit}\left(P_{c, a}^{m}\right)\right) \cup \operatorname{Crit}\left(P_{c, a}^{k}\right) .
$$


Proof. - Write $P=P_{c, a}$ and $Q=Q_{c, a}$. Differentiate the equality $P^{k} \circ Q=Q \circ P^{k}$. Since $Q^{\prime}=\zeta \cdot\left(P^{m}\right)^{\prime}$, we get

$$
\begin{aligned}
\operatorname{Crit}\left(Q \circ P^{k}\right)=P^{-k}(\operatorname{Crit}(Q)) \cup \operatorname{Crit}\left(P^{k}\right) & = \\
& P^{-k}\left(\operatorname{Crit}\left(P^{m}\right)\right) \cup \operatorname{Crit}\left(P^{k}\right)=\operatorname{Crit}\left(P^{k+m}\right),
\end{aligned}
$$

and therefore

$$
\operatorname{Crit}\left(P^{k+m}\right)=\operatorname{Crit}\left(P^{k} \circ Q\right)=\operatorname{Crit}\left(P^{m}\right) \cup Q^{-1}\left(\operatorname{Crit}\left(P^{k}\right)\right),
$$

and we conclude taking the image of both sides by $Q$.

We now come to the proof of the Proposition.

Proof of Proposition 7.3. - We may and shall assume that all objects are defined over the field of complex numbers.

1. Suppose $Z(q, 0, \zeta)$ contains an irreducible curve $C$. We shall prove that either $\zeta= \pm 1$, or $C=\{c=0\}$.

Observe that for any $(c, a) \in Z(q, 0, \zeta)$, the polynomial $Q_{c, a}$ is an affine map which commutes with $P_{c, a}^{k}$, hence $g_{c, a}\left(Q_{c, a}(z)\right)=g_{c, a}(z)$ for all $z \in \mathbb{C}$. Without loss of generality, we may suppose that $Q_{c, a}\left(P_{c, a}^{q}\left(c_{0}\right)\right)=P_{c, a}^{q}\left(c_{1}\right)$, hence $G(c, a)=g_{0}(c, a)=g_{1}(c, a)$.

Suppose that $Z(q, 0, \zeta)$ contains an irreducible curve $C$. If $g_{0}$ vanishes identically on $C$ then $g_{1}$ also, and this implies both critical points to be persistently preperiodic so that all polynomials in $C$ are post-critically finite. This cannot happen, so that we can find an open set $U$ in $C$ such that $G(c, a)>0$ for all $(c, a) \in U$.

Pick any parameter $(c, a)$ in $U$. We have $Q_{c, a}\left(\operatorname{Crit}\left(P_{c, a}^{k}\right)\right)=\operatorname{Crit}\left(P_{c, a}^{k}\right)$ by Lemma 7.4, so that $Q_{c, a}\left(c_{0}\right), Q_{c, a}\left(c_{1}\right) \in \operatorname{Crit}\left(P_{c, a}^{k}\right)$. Since

$$
\operatorname{Crit}\left(P_{c, a}^{k}\right)=\bigcup_{0 \leq j \leq k-1} P_{c, a}^{-j}\left(\operatorname{Crit}\left(P_{c, a}\right)\right)
$$

we get $g_{c, a}(\alpha)=3^{-j} g_{c, a}\left(c_{0}\right)<g_{c, a}\left(c_{0}\right)=G(c, a)$ for any $\alpha$ lying in $\operatorname{Crit}\left(P_{c, a}^{k}\right)$ but not in $\operatorname{Crit}\left(P_{c, a}\right)$. However $g_{c, a}\left(Q_{c, a}\left(c_{0}\right)\right)=g_{c, a}\left(Q_{c, a}\left(c_{0}\right)\right)=G(c, a)$, therefore we have $Q_{c, a}\left(c_{0}\right), Q_{c, a}\left(c_{1}\right) \in \operatorname{Crit}\left(P_{c, a}\right)=\left\{c_{0}, c_{1}\right\}$.

This implies either $(1-\zeta) c=0$, or $(1+\zeta) c=0$, hence $\zeta= \pm 1$ or $C=\{c=0\}$ as required.

2. Suppose now that $C$ is an irreducible curve included in $Z(q, m, \zeta)$ with $m>0$. We claim that either $\zeta=1$, or $C=\{c=0\}$ as above.

We proceed similarly as in the previous case. We suppose that $Z(q, m, \zeta)$ is infinite. For any $(c, a) \in Z(q, m, \zeta)$, the polynomial $Q_{c, a}$ commutes with $P_{c, a}^{k}$ for some $k$, and has degree $3^{m}>1$. In particular we have equality of Green functions $g_{Q_{c, a}}=g_{c, a}$. Without loss of generality we may (and shall) assume $Q_{c, a}\left(P_{c, a}^{q}\left(c_{0}\right)\right)=P_{c, a}^{q}\left(c_{1}\right)$, which implies $g_{0}(c, a)=3^{m} g_{1}(c, a)$.

Assume now by contradiction that $\zeta \neq 1$. Proceeding as in the previous case, we can find an open set $U \subset C$ such that $G(c, a)=g_{0}(c, a)>0$ for all $(c, a) \in U$. Pick now $(c, a) \in U$.

Lemma 7.5. - For any $\alpha \in P_{c, a}^{-m}\left\{c_{0}\right\}$, we have $Q_{c, a}(\alpha) \in\left\{c_{0}, Q_{c, a}\left(c_{1}\right)\right\}$.

Observe that

$$
Q_{c, a}(\alpha)=\zeta P_{c, a}^{m}(\alpha)+(1-\zeta) \frac{c}{2}=\zeta c_{0}+(1-\zeta) \frac{c}{2}=(1-\zeta) \frac{c}{2} .
$$


The equality $Q_{c, a}\left(c_{1}\right)=Q_{c, a}(\alpha)$ therefore gives $Q_{c, a}\left(c_{1}\right)=\zeta P_{c, a}^{m}\left(c_{1}\right)+(1-\zeta) \frac{c}{2}=(1-\zeta) \frac{c}{2}$, and we find $P_{c, a}^{m}\left(c_{1}\right)=0=c_{0}$ so that $C$ is a component of $Z(1, m, 1)$.

The equality $c_{0}=Q_{c, a}(\alpha)$, implies $(1-\zeta) \frac{c}{2}=0$ so that either $\zeta=1$, or $C$ equals $\{c=0\}$.

3. We have $Z(q, 0,-1)=Z(0,0,-1)$ for all $q \geq 0$.

Fix $q \geq 0$, and pick any $(c, a) \in Z(q, 0,-1)$. Observe that $(-1)^{3}=-1$ hence $Q_{c, a}(z)=$ $-z+c$ commutes with $P_{c, a}$ by definition. A direct computation shows that this happens if and only if $(c, a)$ belongs to the curve $D_{1}:=\left\{12 a^{3}-c^{3}-6 c=0\right\}$.

One can also check that $Q_{c, a}\left(c_{0}\right)=c_{1}$ for any parameter on $D_{1}$, and this implies $\left(Q_{c, a} \circ P_{c, a}^{q}\right)\left(c_{0}\right)=P_{c, a}^{q}\left(Q_{c, a}\left(c_{0}\right)\right)=P_{c, a}^{q}\left(c_{1}\right)$ for any $q \geq 0$. This implies the claim.

4. The irreducible curve $D_{0}=\{c=0\}$ is included in $Z(q, m, \zeta)$ if and only if $m=0$ and $\zeta=1$.

Observe that any polynomial $P:=P_{0, a}$ in $D_{0}$ is unicritical with a single critical point at 0 , so that $D_{0}$ is included in $Z(q, 0,1)$ for all $q \geq 0$. Observe also that $g_{0}=g_{1}>0$ on a non-empty open subset of $D_{0}$.

Suppose that $D_{0}$ is included in $Z(q, m, \zeta)$ for some positive integer $m>0$. Then the Green function of $Q:=Q_{0, a}$ is equal to $g_{P}$ and the equation $Q\left(P^{q}\left(c_{0}\right)\right)=P^{q}\left(c_{1}\right)$ implies $g_{P}\left(c_{0}\right)=3^{m} g_{P}\left(c_{1}\right)>g_{P}\left(c_{1}\right)$ at least when $P$ is close enough to infinity. This is absurd.

Suppose now that $D_{0}$ is included in $Z(q, 0, \zeta)$ with $\zeta \neq 1$ so that $Q(z)=\zeta z$. One checks by induction that for any integer $k \geq 1$ one has

$$
P^{k}(z)=q_{k} z^{3^{k}}+s_{k} a^{3} z^{3^{k}-3}+\text { l.o.t }
$$

with $q_{k}, s_{k} \in \mathbb{Q}_{+}^{*}$. Choose $k$ minimal such that $\zeta^{3^{k}}=\zeta$. We get

$$
Q^{-1} \circ P^{k} \circ Q(z)=q_{k} z^{3^{k}}+s_{k} a^{3} \zeta^{3^{k}-4} z^{3^{k}-3}+\text { l.o.t } \neq P^{k}
$$

which yields a contradiction, and concludes the proof of our claim.

5. We may now prove the proposition. The first statement follows from the definition of $Z(q, m, 1)$, since in that case we have $Q=P^{m}$ which always commutes with $P$. Moreover, the curve $Z(1,0,1)$ is given by the equation

$$
0=P_{c, a}\left(c_{0}\right)-P_{c, a}\left(c_{1}\right)=a^{3}-\left(a^{3}-\frac{c^{3}}{6}\right)=\frac{c^{3}}{6},
$$

whence $Z(1,0,1)=\{c=0\}=Z(0,0,1)$.

For the second statement, suppose first that $Z(q, m,-1)$ is infinite. By the second step, we have $m=0$, or $D_{0}=\{c=0\}$ is included in $Z(q, m,-1)$. The fourth step rules out the latter possibility so that $m=0$. Conversely if $m=0$ we may apply the third step to conclude that $Z(q, 0,-1)$ is a curve equal to $D_{1}=\left\{12 a^{3}-c^{3}-6 c=0\right\}$.

For the third statement, pick $\zeta \neq \pm 1$ and suppose by contradiction that $Z(q, m, \zeta)$ is infinite. The first and second step imply that $Z(q, m, \zeta)$ contains $D_{0}$ which is impossible by Step 4 .

This concludes the proof of the proposition.

Proof of Lemma 7.5. - Take $\alpha \in P_{c, a}^{-m}\left\{c_{0}\right\}$, and observe that $\alpha \in \operatorname{Crit}\left(P_{c, a}^{k+m}\right)$. According to Lemma 7.4, we have

$$
Q_{c, a}(\alpha) \in \operatorname{Crit}\left(P_{c, a}^{k}\right) \cup Q_{c, a}\left(\operatorname{Crit}\left(P_{c, a}^{m}\right)\right)
$$

and $g_{c, a}\left(Q_{c, a}(\alpha)\right)=3^{m} g_{c, a}(\alpha)=3^{m} \cdot 3^{-m} g_{c, a}\left(c_{0}\right)=g_{c, a}\left(c_{0}\right)=G(c, a)>0$. 
Pick any point $z \in \operatorname{Crit}\left(P_{c, a}^{k}\right) \cup Q_{c, a}\left(\operatorname{Crit}\left(P_{c, a}^{m}\right)\right)$, and suppose it is equal to neither $c_{0}$ nor $Q_{c, a}\left(c_{1}\right)$. Then we are in one of the following (excluding) cases:

1. $z$ is a preimage of $c_{0}$ under $P_{c, a}^{j}$ for some $1 \leq j \leq k-1$, and $g_{c, a}(z)<g_{P}\left(c_{0}\right)$;

2. $z$ is a preimage of $c_{1}$ under $P_{c, a}^{j}$ for some $0 \leq j \leq k-1$, in which case $g_{c, a}(z) \leq$ $g_{c, a}\left(c_{1}\right)<g_{c, a}\left(c_{0}\right)$

3. $z \in Q_{c, a}\left(\operatorname{Crit}\left(P_{c, a}^{m}\right)\right)$, so that $g_{c, a}(z)=3^{m} g_{c, a}(w)$ for some point $w \in \operatorname{Crit}\left(P_{c, a}^{m}\right)=$ $\bigcup_{0 \leq j \leq m-1} P_{c, a}^{-j}\left(\operatorname{Crit}\left(P_{c, a}\right)\right)$.

In the last case two sub-cases arise. When $w$ is a preimage of $c_{0}$, we find

$$
g_{c, a}(z)=3^{m} g_{c, a}(w) \geq 3^{m} \frac{1}{3^{m-1}} g_{c, a}\left(c_{0}\right)>g_{c, a}\left(c_{0}\right) .
$$

Otherwise $w$ is a preimage of $c_{1}$ distinct from $c_{1}$ since $z \neq Q_{c, a}\left(c_{1}\right)$. And we find $g_{c, a}(z)=$ $3^{m} g_{c, a}(w) \leq 3^{m-1} g_{c, a}\left(c_{1}\right)<g_{c, a}\left(c_{0}\right)$.

Since $g_{c, a}\left(Q_{c, a}(\alpha)\right)=g_{c, a}\left(c_{0}\right)$ we conclude that $z \neq Q_{c, a}(\alpha)$ as required.

\section{References}

[A] Pascal Autissier. Points entiers sur les surfaces arithmétiques. J. Reine Angew. Math., 531:201235, 2001.

[B] Vladimir G. Berkovich. Spectral theory and analytic geometry over non-Archimedean fields, volume 33 of Mathematical Surveys and Monographs. American Mathematical Society, Providence, RI, 1990.

[BB1] Giovanni Bassanelli and François Berteloot. Lyapunov exponents, bifurcation currents and laminations in bifurcation loci. Math. Ann., 345(1):1-23, 2009.

[BB2] Giovanni Bassanelli and François Berteloot. Distribution of polynomials with cycles of a given multiplier. Nagoya Math. J., 201:23-43, 2011.

[BDM] Matthew Baker and Laura De Marco. Special curves and postcritically finite polynomials. Forum Math. Pi, 1:e3, 35, 2013.

[BFJ] Sébastien Boucksom, Charles Favre, and Mattias Jonsson. Singular semipositive metrics in non-Archimedean geometry. J. Algebraic Geom., 25(1):77-139, 2016.

[BH1] M. Baker and L. H. H'sia. Canonical heights, transfinite diameters, and polynomial dynamics. J. Reine Angew. Math, (585):61-92, 2005.

[BH2] Bodil Branner and John Hamal Hubbard. The iteration of cubic polynomials. I. The global topology of parameter space. Acta Math., 160(3-4):143-206, 1988.

[BKM] Araceli Bonifant, Jan Kiwi, and John Milnor. Cubic polynomial maps with periodic critical orbit. II. Escape regions. Conform. Geom. Dyn., 14:68-112, 2010.

[C] Evgenii Mikhailovich Chirka. Complex analytic sets, volume 46 of Mathematics and its Applications (Soviet Series). Kluwer Academic Publishers Group, Dordrecht, 1989. Translated from the Russian by R. A. M. Hoksbergen.

[CL1] Antoine Chambert-Loir. Mesures et équidistribution sur les espaces de Berkovich. J. Reine Angew. Math., 595:215-235, 2006.

[CL2] Antoine Chambert-Loir. Heights and measures on analytic spaces. A survey of recent results, and some remarks. In Motivic integration and its interactions with model theory and nonArchimedean geometry. Volume II, volume 384 of London Math. Soc. Lecture Note Ser., pages 1-50. Cambridge Univ. Press, Cambridge, 2011.

[De1] Laura DeMarco. Dynamics of rational maps: a current on the bifurcation locus. Math. Res. Lett., 8(1-2):57-66, 2001.

[De2] Laura DeMarco. Bifurcations, intersections, and heights, 2014. preprint arXiv:1412.2708. 
[Du1] Romain Dujardin. Cubic polynomials: a measurable view on parameter space. In Complex dynamics : families and friends / edited by Dierk Schleicher, pages 451-490. A K Peters, Ltd., Wellesley, Massachussets, 2009.

[Du2] Romain Dujardin. The supports of higher bifurcation currents. Ann. Fac. Sci. Toulouse Math. (6), 22(3):445-464, 2013.

[DF] Romain Dujardin and Charles Favre. Distribution of rational maps with a preperiodic critical point. Amer. J. Math., 130(4):979-1032, 2008.

[DH] Adrien Douady and John Hamal Hubbard. Étude dynamique des polynômes complexes. Partie I, volume 84 of Publications Mathématiques d'Orsay [Mathematical Publications of Orsay]. Université de Paris-Sud, Département de Mathématiques, Orsay, 1984.

[DMS] Laura De Marco and Aaron Schiff. The geometry of the critically periodic curves in the space of cubic polynomials. Exp. Math., 22(1):99-111, 2013.

[DMWY] Laura De Marco, Xiaoguang Wang, and Hexi Ye. Bifurcation measures and quadratic rational maps. Proc. Lond. Math. Soc. (3), 111(1):149-180, 2015.

[FG] Charles Favre and Thomas Gauthier. Distribution of postcritically finite polynomials. Israel J. Math., 209(1):235-292, 2015.

[FRL] Charles Favre and Juan Rivera-Letelier. Théorie ergodique des fractions rationnelles sur un corps ultramétrique. Proc. Lond. Math. Soc. (3), 100(1):116-154, 2010.

[G1] Thomas Gauthier. Strong bifurcation loci of full Hausdorff dimension. Ann. Sci. Éc. Norm. Supér. (4), 45(6):947-984, 2012.

[G2] Thomas Gauthier. Equidistribution towards the bifurcation current I: Multipliers and degree $d$ polynomials, 2013. to appear in Math. Ann.

[G3] Thomas Gauthier. Higher bifurcation currents, neutral cycles, and the Mandelbrot set. Indiana Univ. Math. J., 63(4):917-937, 2014.

[GV] Thomas Gauthier and Gabriel Vigny. Distribution of postcritically finite polynomials ii: Speed of convergence, 2015. preprint arXiv:1505.07325.

[H1] Lars Hörmander. An introduction to complex analysis in several variables, volume 7 of NorthHolland Mathematical Library. North-Holland Publishing Co., Amsterdam, third edition, 1990.

[H2] Lars Hörmander. Notions of convexity. Modern Birkhäuser Classics. Birkhäuser Boston, Inc., Boston, MA, 2007. Reprint of the 1994 edition.

[I] Patrick Ingram. A finiteness result for post-critically finite polynomials. Int. Math. Res. Not. IMRN, (3):524-543, 2012.

[J] Gaston Julia. Mémoire sur la permutabilité des fractions rationnelles. Ann. Sci. École Norm. Sup. (3), 39:131-215, 1922.

[K] Jan Kiwi. Puiseux series polynomial dynamics and iteration of complex cubic polynomials. Ann. Inst. Fourier (Grenoble), 56(5):1337-1404, 2006.

[L] Genadi Levin. On the theory of iterations of polynomial families in the complex plane. J. Soviet Math., 52(6):3512-3522, 1990.

[McM1] Curtis McMullen. Families of rational maps and iterative root-finding algorithms. Ann. of Math. (2), 125(3):467-493, 1987.

[McM2] Curtis T. McMullen. Complex dynamics and renormalization, volume 135 of Annals of Mathematics Studies. Princeton University Press, Princeton, NJ, 1994.

[McM3] Curtis T. McMullen. The Mandelbrot set is universal. In The Mandelbrot set, theme and variations, volume 274 of London Math. Soc. Lecture Note Ser., pages 1-17. Cambridge Univ. Press, Cambridge, 2000.

[Mi1] John Milnor. Geometry and dynamics of quadratic rational maps. Experiment. Math., 2(1):37-83, 1993. With an appendix by the author and Lei Tan.

[Mi2] John Milnor. Dynamics in one complex variable, volume 160 of Annals of Mathematics Studies. Princeton University Press, Princeton, NJ, third edition, 2006. 
[Mi3] John Milnor. Cubic polynomial maps with periodic critical orbit. I. In Complex dynamics, pages 333-411. A K Peters, Wellesley, MA, 2009.

[MS] Alice Medvedev and Thomas Scanlon. Invariant varieties for polynomial dynamical systems. Ann. of Math. (2), 179(1):81-177, 2014.

$[\mathrm{P}]$ Fedor Pakovich. Polynomial semiconjugacies, decompositions of iterations, and invariant curves, 2015. preprint arXiv:1505.06351.

[Se] Jean-Pierre Serre. Lie algebras and Lie groups, volume 1500 of Lecture Notes in Mathematics. Springer-Verlag, Berlin, 2006. 1964 lectures given at Harvard University, Corrected fifth printing of the second (1992) edition.

[Si1] Joseph H. Silverman. The arithmetic of dynamical systems, volume 241 of Graduate Texts in Mathematics. Springer, New York, 2007.

[Si2] Joseph H. Silverman. Moduli spaces and arithmetic dynamics, volume 30 of CRM Monograph Series. American Mathematical Society, Providence, RI, 2012.

[T] Amaury Thuillier. Théorie du potentiel sur les courbes en géométrie analytique non archimédienne. applications à la théorie d'arakelov, 2005. Thèse de l'Université de Rennes 1, viii $+184 \mathrm{p}$.

[X] Junyi Xie. Intersections of valuation rings in $k[x, y]$. Proc. Lond. Math. Soc. (3), 111(1):240$274,2015$.

[Y] Xinyi Yuan. Big line bundles over arithmetic varieties. Invent. Math., 173(3):603-649, 2008.

[Z] Shou-Wu Zhang. Positive line bundles on arithmetic varieties. J. Amer. Math. Soc., 8:187-221, 1995.

September 12, 2018

Charles Favre, CMLS, École polytechnique, CNRS, Université Paris-Saclay, 91128 Palaiseau Cedex, France - E-mail : charles.favre@polytechnique.edu

Thomas Gauthier, LAMFA, Université de Picardie Jules Verne, 33 rue Saint Leu, 80039 Amiens Cedex E-mail : thomas.gauthier@u-picardie.fr 